\title{
4-DIMENSIONAL SYMPLECTIC CONTRACTIONS
}

\author{
MARCO ANDREATTA AND JAROSŁAW A. WIŚNIEWSKI
}

\begin{abstract}
Local symplectic contractions are resolutions of singularities which admit symplectic forms. Four dimensional symplectic contractions are (relative) Mori Dream Spaces. In particular, any two such resolutions of a given singularity are connected by a sequence of Mukai flops. We discuss the cone of movable divisors on such a resolution; its faces are determined by curves whose loci are divisors, we call them essential curves. The movable cone is divided into nef chambers which are related to different resolutions; this subdivision is determined by classes of 1-cycles. We also study schemes parametrizing minimal essential curves and show that they are resolutions, possibly nonminimal, of surface Du Val singularities. Some examples, with an exhaustive description, are provided.
\end{abstract}

\section{INTRODUCTION}

In the paper we consider local symplectic contractions of 4 -folds. That is, we deal with maps $\pi: X \rightarrow Y$ where

- $X$ is a smooth complex 4-fold with a closed holomorphic 2-form, nondegenerate at every point,

- $Y$ is an affine (or Stein) normal variety,

- $\pi$ is a birational projective morphism.

In dimension 2 symplectic contractions are classical and they are minimal resolutions of Du Val singularities. In fact, any symplectic contraction can be viewed as a special symplectic resolution of a symplectic normal singularity.

General properties of symplectic contractions (in arbitrary dimension) have been considered in a number of papers published in the last decade: Bea00, Ver00, Nam01, Kal02, Kal03, Wie03, [FN04, GK04, Fu06a, [HT09, Bel09, [LS08], to mention just a few; see also [Fu06b] for more references and a review on earlier developments in this subject. Let us just recall two beautiful results about symplectic contractions: these maps are semismall, Wie03, and the McKay correspondence holds for those symplectic contractions which are resolutions of quotient symplectic singularities, Kal02, GK04. However, in dimension 4 and higher, apart from the description in codimension 2, Wie03, not much is known about the fine geometrical structure of these morphisms which is the problem we want to tackle in the present paper.

The 4-dimensional small case (i.e. when $\pi$ does not contract a divisor) is known by WW03, Thm. 1.1]. Using this result we first prove in Section 3.1 that $X$ is a Mori

1991 Mathematics Subject Classification. 20C10, 14E15, 14F10, 14J28, 14J32.

The first author was supported by the Italian PRIN; he thanks also the Institute of Mathematics of Warsaw University for hospitality. The second author was supported by Polish MNiSzW grants N N201 265333 and N N201 420639 as well as Fondazione Bruno Kessler at CIRM in Trento. Thanks to Andrzej Weber for stimulating discussions. 
Dream Space over $Y$, as defined in [HK00]. In short, every movable divisor of $X$ (over $Y$ ) can be made nef and semiample after a finite number of small modifications (flops); see also [Wie02] and [WW03. Thm. 1.2], where a version of this result was announced. In Theorem 4.1 we describe the cone of movable divisors of $X / Y$; by Theorem 3.5 it can be also described in terms of classes of curves contracted in the divisorial locus. As a result, these objects are (with one possible exception) as in the case of the contraction to the nilpotent cone (we recall this situation in the Appendix). Next, we give properties of the subdivision of the cone of movable divisors into chambers corresponding to nef cones of small modifications of $X$, see Theorem 3.3 .

In Section 5. following the approach introduced in Wie03 and subsequently in SCW04, we study families of rational curves (i.e. irreducible components of the Chow scheme) in $X / Y$. In Theorem 5.1 we prove that they are resolutions of $\mathrm{Du}$ Val singularities, possibly non-minimal; the discrepancies of this resolution depend on the rank of the evaluation map, see Proposition 5.2 and on the modification of $X$, see Lemma 6.5. We also show that studying 4-dimensional symplectic resolutions implies understanding arbitrary dimensional case in codimension 4, via the argument of general intersection of a suitable number of divisors, we call it vertical slicing, see Corollary 5.4

In Section 6 we study known examples of resolutions of quotient symplectic singularities and we describe explicitly their movable cones and their families of rational curves. In particular, we describe explicitly the division of the movable cone of a symplectic resolution of $\mathbb{C}^{4} /\left(\mathbb{Z}_{n+1} \prec \mathbb{Z}_{2}\right)$ into nef cones which are associated with different resolutions of this singularity. The main result of this section, Theorem 6.6. gives a description of the cone of movable divisors into nef chambers by hyperplanes defined by classes of contracted curves contained in the divisorial locus. The following Theorem summarizes our results in this particular case and, in fact, serves as a model of the situation that we expect to get while dealing with arbitrary local symplectic contraction in dimension 4 (for definitions and other examples see Section 4).

Theorem 1.1. Let $\mathbb{A}_{1} \oplus \mathbb{A}_{n}$ be a decomposable root system in $V \simeq \mathbb{R}^{n+1}$ with simple (positive) roots denoted by $e_{0}$ and $e_{1}, \ldots, e_{n}$. Let $\mathrm{Mov}=\left\langle e_{0}, e_{1}, \ldots, e_{n}\right\rangle \vee$ be the cone in $V^{\vee}$ dual to the cone spanned by the roots $e_{0}, e_{1}, \ldots, e_{n}$. Then the symplectic resolutions of the singularity $\mathbb{C}^{4} /\left(\mathbb{Z}_{n+1}<\mathbb{Z}_{2}\right)$ are in bijective relation with maximal dimensional cones obtained by cutting the cone Mov with hyperplanes $\left(e_{0}-e_{i j}\right)^{\perp}$ where vectors $e_{i j}=e_{i}+\cdots+e_{j}$, for $1 \leq i \leq j \leq n$, are all positive roots of the root system $\mathbb{A}_{n}$.

\section{NotATION AND PRELIMINARIES}

2.1. Symplectic contractions. A holomorphic 2 -form $\omega$ on a smooth variety is called symplectic if it is closed and non-degenerate at every point. A symplectic variety is a normal variety $Y$ whose smooth part admits a holomorphic symplectic form $\omega_{Y}$ such that its pull back to any resolution $\pi: X \rightarrow Y$ extends to a holomorphic 2-form $\omega_{X}$ on $X$. We call $\pi$ a symplectic resolution if $\omega_{X}$ is non degenerate on $X$, i.e. it is a symplectic form. More generally, a map $\pi: X \rightarrow Y$ is called a symplectic contraction if $X$ is a symplectic manifold, $Y$ is normal and $\pi$ is a birational projective morphism. If moreover $Y$ is affine we will call $\pi: X \rightarrow Y$ a 
local symplectic contraction or local symplectic resolution. The following facts are well known, see the survey paper [Fu06b] and references therein.

Proposition 2.1. Let $Y$ be a symplectic variety and $\pi: X \rightarrow Y$ be a resolution. Then the following statement are equivalent: (i) $\pi^{*} K_{Y}=K_{X}$, (ii) $\pi$ is symplectic, (iii) $K_{X}$ is trivial, (iv) for every symplectic form on $Y_{\text {reg }}$ its pull-back extends to a symplectic form on $X$.

Theorem 2.2. A symplectic resolution $\pi: X \rightarrow Y$ is semismall, that is for every closed subvariety $Z \subset X$ we have $2 \operatorname{codim} Z \geq \operatorname{codim} \pi(Z)$. If equality holds $Z$ then is called a maximal cycle.

Corollary 2.3. Any two symplectic resolutions $\pi_{i}: X_{i} \rightarrow Y$, where $i=1,2$, are isomorphic in codimension 1.

Proof. By 2.2 every exceptional divisor of a symplectic resolution $\pi_{i}$ is mapped to a codimension 2 set in $Y$. On the other hand, any symplectic resolution of $Y$ is uniquely determined in codimension 2 as the resolution of the surface Du Val singularities.

Example 2.4. Let $S$ be a smooth surface (proper or not). Denote by $S^{(n)}$ the symmetric product of $S$, that is $S^{(n)}=S^{n} / \sigma_{n}$, where $\sigma_{n}$ is the symmetric group of peremutations of $n$ elements. Let also $\operatorname{Hilb}^{n}(S)$ be the Hilbert scheme of 0-cycles of degree $n$. A classical result (c.f. Fog68) says that $\operatorname{Hilb}^{n}(S)$ is smooth and that $\tau: \operatorname{Hilb}^{n}(S) \rightarrow S^{(n)}$ is a crepant resolution of singularities. We will call it a Hilb-Chow map.

Suppose now that $S \rightarrow S^{\prime}$ is a resolution of a Du Val singularity which is of type $S^{\prime}=\mathbb{C}^{2} / H$ with $H<S L(2, \mathbb{C})$ a finite group. Then the composition $H_{i l b}(S) \rightarrow$ $S^{(n)} \rightarrow\left(S^{\prime}\right)^{(n)}$ is a local symplectic contraction.

We note that $\left(S^{\prime}\right)^{(n)}$ is a quotient singularity with respect to the action of the wreath product $H \imath \sigma_{n}=\left(H^{n}\right) \rtimes \sigma_{n}$ (the group $\sigma_{n}$ permutes factors in $\left.H^{n}=H^{\times n}\right)$.

2.2. Mori Dream Spaces. Let us recall basic definitions regarding Mori Dream Spaces. For more information we refer to [HK00] or [ADHL10. Our definitions are far from general but sufficient for our particular local set-up. We assume that

(1) $\pi: X \rightarrow Y$ is a projective morphism of normal varieties with connected fibers, that is $\pi_{*} \mathcal{O}_{X}=\mathcal{O}_{Y}$ and $Y=\operatorname{Spec} A$ is affine,

(2) $X$ is locally factorial and $\operatorname{Pic}(X / Y)=\mathrm{Cl}(X / Y)$ is a finitely generated abelian group so that $N^{1}(X / Y)=\operatorname{Pic}(X / Y) \otimes \mathbb{Q}$ is a finite dimensional vector space

By $\operatorname{Nef}(X / Y) \subset N^{1}(X / Y)$ we understand the closure of the convex cone spanned by the classes of relatively ample bundles, while by $\operatorname{Mov}(X / Y) \subset N^{1}(X / Y)$ we understand the closure of the convex cone spanned by the classes of linear systems which have no fixed components. That is, a class of a $\mathbb{Q}$-divisor $D$ is in $\operatorname{Mov}(X / Y)$ if the linear system $|m D|$ has no fixed component for $m \gg 0$. Clearly $\operatorname{Nef}(X / Y) \subset$ $\operatorname{Mov}(X / Y)$ and we call $\operatorname{Nef}(X / Y)$ the Mori cone (or chamber) of $X$. By the relative version of Kleiman's criterion of ampleness the cone $\operatorname{Nef}(X / Y)$ is strictly convex. The following is a version of [HK00, Def. 1.10].

Definition 2.5. In the above situation we say that $X$ is a Mori Dream Space (MDS) over $Y$ if in addition 
(1) the cone $\operatorname{Nef}(X / Y)$ is spanned by the classes of finitely many semi-ample line bundles:

(2) there is a finite collection of small $\mathbb{Q}$-factorial modifications (SQM) over $Y$, $f_{i}: X-\rightarrow X_{i}$ such that $X_{i} \longrightarrow Y$ satisfies the above assumptions and $\operatorname{Mov}(X / Y)$ is the union of the strict transforms $\left(f_{i}\right)_{*}\left(\operatorname{Nef}\left(X_{i} / Y\right)\right)$

Here, since $X$ and $X_{i}$ are both $\mathbb{Q}$-factorial and isomorphic in codimension 1 , we can identify, via $f_{i}, N^{1}(X / Y)$ with $N^{1}\left(X_{i} / Y\right)$ and $\operatorname{Mov}(X / Y)$ with $\operatorname{Mov}\left(X_{i} / Y\right)$. By abuse we will just write $N^{1}(X / Y)=N^{1}\left(X_{i} / Y\right)$ and $\operatorname{Mov}(X / Y)=\operatorname{Mov}\left(X_{i} / Y\right)$.

Example 2.6. Take the $\mathbb{C}^{*}$ action on $\mathbb{C}^{r} \times \mathbb{C}^{r}$ with coordinates $\left(x_{i}, y_{j}\right)$ and weights 1 for $x_{i}$ 's and -1 for $y_{j}$ 's. Using these weights we define a $\mathbb{Z}$-grading of the polynomial ring and write $\mathbb{C}\left[x_{i}, y_{j}\right]=\bigoplus_{m \in \mathbb{Z}} A_{m}$. The quotient $\widehat{Y}=\operatorname{Spec} A_{0}$ is a toric singularity which, in the language of toric geometry, is associated with a cone spanned by $r$ vectors $e_{i}$ and $f_{j}$, in the lattice of rank $2 r-1$, with one relation $\sum e_{i}=\sum f_{j}$. The result is the cone over Segre embedding of $\mathbb{P}^{r-1} \times \mathbb{P}^{r-1}$. Consider $A_{+}=\bigoplus_{m \geq 0} A_{m}$ and $A_{-}=\bigoplus_{m \leq 0} A_{m}$, and define two varieties over $\widehat{Y}$ :

$$
\widehat{X}_{ \pm}=\operatorname{Proj}_{A_{0}} A_{ \pm} \longrightarrow \widehat{Y}
$$

Both, $\widehat{X}_{+}$and $\widehat{X}_{-}$, are smooth because, as toric varieties, they are associated with two unimodular triangulations of the cone in question: one in which we omit consecutive $e_{i}$ 's, the other in which we omit $f_{j}$ 's. The affine pieces of covering are of type $\operatorname{Spec} \mathbb{C}\left[x_{i} / x_{k}, x_{k} y_{j}\right]$, where $k=1, \ldots, r$, for $\widehat{X}_{+}$and similar for $\widehat{X}_{-}$. The two resolution $\widehat{X}_{+} \rightarrow \widehat{Y} \leftarrow \widehat{X}_{-}$form two sides of the so-called Atiyah flop.

Consider an ideal $I=\left(\sum_{i} x_{i} y_{i}\right) \triangleleft \mathbb{C}\left[x_{i}, y_{j}\right]$ generated by a $\mathbb{C}^{*}$ invariant function (degree 0 ) and its respective counterparts $I_{0} \cap A_{0} \triangleleft A_{0}, I_{+} \triangleleft A_{+}$and $I_{-} \triangleleft A_{-}$. We set $Y=\operatorname{Spec} A_{0} / I_{0}, X_{+}=\operatorname{Proj}_{A_{0}} A_{+} / I_{+}$and $X_{-}=\operatorname{Proj}_{A_{0}} A_{-} / I_{-}$and call the resulting diagram $X_{+} \rightarrow Y \leftarrow X_{-}$Mukai flop. By abuse, by the same name we will call a respective diagram which is locally isomorphic to the present one in the analytic or formal category. The variety $Y$ is symplectic since the form $\omega=\sum_{i}\left(d x_{i} \wedge d y_{i}\right)$ on $\mathbb{C}^{r} \times \mathbb{C}^{r}$ descends to a symplectic form on $Y$. The varieties $X_{ \pm}$are its small symplectic resolutions and $\mathbb{C}\left[x_{i}, y_{j}\right] / I$ is their Cox ring, ADHL10. We note that $\operatorname{Spec}\left(\mathbb{C}\left[x_{i}, y_{j}\right] / I\right)$ is the cone over the incidence variety of points and hyperplanes in $\mathbb{P}^{r-1} \times\left(\mathbb{P}^{r-1}\right)^{*}$. Finally, we note that the movable cone $\operatorname{Mov}\left(X_{ \pm}\right)$ is the whole line $N^{1}\left(X_{ \pm}\right)$, hence it is not strictly convex.

\section{LOCAL SYMPLECTIC CONTRACTIONS IN DIMENSION 4.}

3.1. MDS structure. In this section $\pi: X \rightarrow Y$ is a local symplectic contraction, as defined in 2.1, and $\operatorname{dim} X=4$. By the semismall property (see Theorem 2.2), the fibers of $\pi$ have dimension less or equal to 2 . We will denote with 0 the unique (up to shrinking $Y$ to a smaller affine set) point such that $\operatorname{dim} \pi^{-1}(0)=2$. If $\pi$ is divisorial then the general non trivial fiber has dimension 1.

We note that $\pi: X \rightarrow Y$ satisfies the assumptions stated in 2.2. In particular, because $R^{i} \pi_{*} \mathcal{O}_{X}=0$ for $i>0$, it follows that $N^{1}(X / Y)$ is a finite dimensional vector space. By $N_{1}(X / Y)$ we denote the $\mathbb{Q}$ vector space of 1-cycles proper over $Y$, modulo numerical equivalence (c.f. KoMo98, Ex. 2.16]). Then $N_{1}(X / Y)$ and $N^{1}(X / Y)$ are dual via the intersection pairing.

We start by recalling the following theorem from WW03, Thm. 1.1]. 
Theorem 3.1. Suppose that $\pi$ is small (i.e. it does not contract any divisor). Then $\pi$ is locally analytically isomorphic to the collapsing of the zero section in the cotangent bundle of $\mathbb{P}^{2}$. Therefore $X$ admits a Mukai flop as described in example 2.6

The above theorem, together with Matsuki's termination of 4-dimensional flops, see Mat91, is the key ingredient in the proof of the following result. Similar results are [WW03, Thm. 1.2], [FN04, Thm. 4.1], and in Wie02, as well as in BHL03. The classical references for the Minimal Model Program (MMP), which is the framework for this argument, are KMM87 and KoMo98; the finitness argument is in KaMa87.

Theorem 3.2. Let $\pi: X \rightarrow Y$ be a 4-dimensional local symplectic contraction and let $\pi^{-1}(0)$ be its only 2-dimensional fiber. Then $X$ is a Mori Dream Space over $Y$. Moreover any SQM model of $X$ over $Y$ is smooth and any two of them are connected by a finite sequence of Mukai flops whose centers are over $0 \in Y$. In particular, there are only finitely many non isomorphic (local) symplectic resolutions of $Y$, c.f. [FN04, Thm. 4.1].

Proof. We note that, by the (relative) non-vanishing theorem (Theorem 3.4 in KoMo98), the linear and numerical equivalence over $Y$ are the same hence $\operatorname{Pic}(X / Y)$ is a finitely generated free abelian group (a lattice). Note also that, since we are in a relative situation over $Y$ via a birational map $\pi$, we can assume that a $\pi$-nef line bundle is also $\pi$-big: in fact a nef bundle plus a big one is big and in our situation the trivial bundle is $\pi$-big. By the (relative) Kawamata-Shokurov basepoint-free theorem (Theorem 3.24 in KoMo98) every nef divisor on $X$ (we drop from now on the suffix $\pi$ ) is also semiample. On the other hand, the (relative) rationality theorem (we use Theorem 3.25(2) in KoMo98, we choose an effective $\mathbb{Q}$-divisor $\Delta$ such that $-\Delta$ is $\pi$-ample which is possible by Kodaira's lemma) asserts that $\operatorname{Nef}(X / Y)$ is rational polyhedral.

Next we claim that $X$ satisfies the second property of definition 2.5. To achieve this, first we prove that for any $\pi_{i}: X_{i} \rightarrow Y$ which is a small $\mathbb{Q}$-factorial modification of $X$, there exists a finite number of Mukai flops $X-\rightarrow \cdots-\rightarrow X_{i}$ which modifies $X$ to $X_{i}$. For this, we take $D_{i}$ which ample on $X_{i}$ and its strict transform is a movable divisor on $X$. We may assume that $X$ is not isomorphic (over $Y$ ) to $X_{i}$ hence $D_{i}$ is not nef. We look for extremal rays which have negative intersection with it. They have to be associated with small contractions because they have to be in the base point locus of the divisor. By theorem 3.1 these are contractions of a $\mathbb{P}^{2}$ which can be flopped (via a Mukai flop) so that the result remains smooth. The process has to finish by the "termination of flops" (relative to the chosen movable divisor), which is the main result in Mat91. Therefore, after a finite number of flops, over a variety $X_{i}^{\prime}$ the strict transform of $D_{i}$ becomes a nef divisor, which is semiample (by the basepoint-free Theorem 3.24 in [KoMo98]). The induced regular morphism

$$
X_{i}^{\prime} \rightarrow \operatorname{Proj}_{Y}\left(\bigoplus_{m \geq 0} \Gamma\left(X_{i}^{\prime}, \mathcal{O}\left(m D_{i}\right)\right)\right)=X_{i}
$$

is in fact an isomorphism because it induces an isomorphism $N^{1}\left(X_{i}^{\prime} / Y\right)=N^{1}\left(X_{i} / Y\right)$. It remains to prove that the subdivision of $\operatorname{Mov}(X / Y)$ into the nef subcones of different SQM's is finite. The argument is the same as in the proof of the main theorem of KaMa87, pp. 596 - 597, where we refer the reader for details. Namely, 
we choose an effective $\mathbb{Q}$ divisor $\Delta$ such that $-\Delta$ is $\pi$-ample and the pair $(X, \Delta)$ is Kawamata log-terminal, or klt. In fact the pair $\left(X_{i}, \Delta_{i}\right)$ is klt for any small $\mathbb{Q}$-factorial modification of $X, \pi_{i}: X_{i} \rightarrow Y$ with $\Delta_{i}$ the strict transform of $\Delta$. By Kawamata rationality theorem, see [KMM87, Thm. 4-1-1] or [KoMo98, Thm. 3.5], the nef threshold for any ample divisor on $X_{i}$ has a bounded denominator which is impossible if the number of Mori chambers in $\operatorname{Mov}(X / Y)$ is infinite.

3.2. Flopping classes. The following theorem provides a somewhat more refined description of the division of the cone $\operatorname{Mov}(X / Y)$.

Theorem 3.3. Let $\pi: X \rightarrow Y$ be a local 4-dimensional symplectic contraction. The subdivision of $\operatorname{Mov}(X / Y)$ into the nef subcones of different SQM models is obtained by cutting $\operatorname{Mov}(X / Y)$ with hyperplanes. That is, the union of the interiors of nef cones of all SQM models of $X$ is equal to $\operatorname{Mov}(X / Y) \backslash \bigcup \lambda_{i}^{\perp}$, where $\lambda_{i}$ 's are classes in $N_{1}(X / Y)$. Moreover the number of these hyperplanes is finite as the number of the chambers is finite as well.

The $\lambda_{i}$ 's in the above theorem are determined up to multiplicity and they will be called the flopping classes of $X$. We think about $\lambda_{i}$ 's as vectors in the dual of $N^{1}(X / Y)$, which is $N_{1}(X / Y)$, supporting hyperplanes $\lambda_{i}^{\perp}=\left\{v \in N^{1}(X / Y)\right.$ : $\lambda \cdot v=0\}$. We assume that $\lambda_{i}^{\perp}$ have non-emty intersection with the interior of the cone $\operatorname{Mov}(X / Y)$. If $\pi^{\prime}: X^{\prime} \rightarrow Y$ is a small $\mathbb{Q}$-factorial modification of $X$ then, via the identification $N^{1}(X / Y)=N^{1}\left(X^{\prime} / Y\right)$, the hyperplanes $\lambda_{i}^{\perp}$ 's are well defined for $X^{\prime}$ and we call them the flopping classes of $X^{\prime}$ as well. We note, however, that there is no natural identification of 1-cycles in $X$ and $X^{\prime}$.

Proof. Take a cone $F$ which is a face the nef cone of some SQM, say $X$, in the interior of $\operatorname{Mov}(X / Y)$. Thus the exceptional locus of the contraction of $X$ associated with the face $F$ consists of a number of disjoint copies of $\mathbb{P}^{2}$. This statement requires not only the theorem 3.1 but also the argument proving that $\mathbb{P}^{2}$ 's are disjoint; the latter is in the proof of (3.2) of WW03.

Let $W=\lambda^{\perp} \cap \operatorname{Nef}(X / Y) \supset F$ be a facet (maximal dimensional face) of $\operatorname{Nef}(X / Y)$ which is also a wall of the subdivision of $\operatorname{Mov}(X / Y)$ into nef chambers associated to different SQM's. The class $\lambda \in N_{1}(X / Y)$ can be realized by rational curves contracted by an extremal contraction of $X$ which factors the contraction associated to $F$.

If $W^{\prime}$ is another facet of $\operatorname{Nef}(X / Y)$ containing $F$ then loci of curves determining $W$ and $W^{\prime}$ are disjoint. Thus the flop $X-\rightarrow X^{\prime}$ with respect to the wall $W^{\prime}$ does not affect curves whose class is $\lambda$. That is, $W=\lambda^{\perp} \subset N^{1}\left(X^{\prime} / Y\right)$ is determined by the class of a curve. This will remain true for any further flop associated to any wall containing the cone $F$. Thus, as a dividing wall in $\operatorname{Mov}(X / Y), W=\lambda^{\perp}$ extends to a hyperplane around $F$.

One might expect that the subdivision of $\operatorname{Mov}(X / Y)$ by the flopping clases can be related to the 2-dimensional components of the central fiber $\pi^{-1}(0)$ of the 4dimensional symplectic contraction $\pi: X \rightarrow Y$. We note that, if $\pi^{\prime}: X^{\prime} \rightarrow Y$ is obtained from $X$ via a Mukai flop $X-\rightarrow X^{\prime}$, then there is a natural bijection between 2-dimensional components of $\pi^{-1}(0)$ and of $\pi^{\prime-1}(0)$. Indeed, a Mukai flop exchanges a number of $\mathbb{P}^{2}$ 's into their "opposite" $\mathbb{P}^{2}$ 's, while on the other components it is a composition of blow-ups and blow-downs. It is not clear however 
if, after a sequence of Mukai flops, a given component of $\pi^{-1}(0)$ may become the locus of a different flopping classes.

3.3. Essential curves. The following definition of essential curves is a simplified version of the one introduced in [AlWi13, suitable for the present set-up.

Definition 3.4. Let $\pi: X \rightarrow Y$ be a 4-dimensional local symplectic contraction with the unique 2-dimensional fiber $\pi^{-1}(0)$. Recall that by $N_{1}(X / Y)$ we denote the $\mathbb{Q}$-vector space of 1 -cycles proper over $Y$. We define $\operatorname{Ess}(X / Y)$ as the convex cone spanned by the classes of curves which are not contained in $\pi^{-1}(0)$. Classes of curves in $\operatorname{Ess}(X / Y)$ are called essential curves.

Theorem 3.5. (c.f. AlWi13) The cones $\operatorname{Mov}(X / Y)$ and $\operatorname{Ess}(X / Y)$ are dual in terms of the intersection product of $N^{1}(X / Y)$ and $N_{1}(X / Y)$, that is $\operatorname{Mov}(X / Y)=$ $\operatorname{Ess}(X / Y)^{\vee}$.

Proof. First we note that if $D$ is a movable divisor on $X$, or if $|m D|$ has no fixed component for $m \gg 0$, then the base point locus of $|m D|$ is contained in $\pi^{-1}(0)$. This yields the obvious inclusion $\operatorname{Mov}(X / Y) \subseteq \operatorname{Ess}(X / Y)^{\vee}$. Moreover, since by 3.2 any two symplectic resolutions of $Y$ are connected by a sequence of flops in centers over $0 \in Y$, it follows that the intersection of divisors with curves outside $\pi^{-1}(0)$ does not depend on the choice of the resolution (or SQM of $X$ ). That is, if $C \subset X \backslash \pi^{-1}(0)$ is a curve proper over $Y, \pi^{\prime}: X^{\prime} \rightarrow Y$ another symplectic resolution and $D^{\prime}$, the strict transform of $D$, then $D \cdot C=D^{\prime} \cdot C$.

Now assume by contradiction that $\operatorname{Mov}(X / Y) \neq \operatorname{Ess}(X / Y)^{\vee}$. Let $F$ be a facet, i.e. a codimension 1 face of $\operatorname{Mov}(X / Y)$. Since $X$ is MDS, we can take a resolution $\pi^{\prime}$ : $X^{\prime} \rightarrow Y$, for which $F \cap \operatorname{Nef}\left(X^{\prime} / Y\right)$ is an extremal face of $\operatorname{Nef}\left(X^{\prime} / Y\right)$. The relative elementary contraction $X^{\prime} \rightarrow Y^{\prime} \rightarrow Y$ of the face $F \cap \operatorname{Nef}\left(X^{\prime} / Y\right)$ is divisorial. Indeed, if it is not, then after a flop we would get another $X^{\prime \prime} \rightarrow Y$ whose nef cone $\operatorname{Nef}\left(X^{\prime \prime} / Y\right)$ is on the other side of the face $F \cap \operatorname{Nef}\left(X^{\prime} / Y\right)$, contradicting the fact that $F$ is an extremal face of $\operatorname{Mov}(X / Y)$. Now we can choose a curve $C \subset X^{\prime} \backslash\left(\pi^{\prime}\right)^{-1}(0)=X \backslash \pi^{-1}(0)$ contracted by $X^{\prime} \rightarrow Y^{\prime}$ and we get the inclusion $F \subset C^{\perp}$. Thus every facet of $\operatorname{Mov}(X / Y)$ is supported by a curve in $\operatorname{Ess}(X / Y)$, hence $\operatorname{Mov}(X / Y)^{\vee} \subseteq \operatorname{Ess}(X / Y)$ and we are done.

From the proof it follows that the above result remains true also if $\pi: X \rightarrow Y$ is a higher dimensional symplectic contraction and $X$ is MDS over $Y$, and essential curves are defined as those whose loci are in codimension 1.

We note that, since the map $\pi$ is assumed to be projective, the cone $\operatorname{Nef}(X / Y)$, hence also the cone $\operatorname{Mov}(X / Y)$, is of maximal dimension. On the other hand we have the following observation.

Proposition 3.6. Let $\pi: X \rightarrow Y$ be as in Theorem 3.2 (or, more generally, suppose that $X$ is a $M D S$ over $Y$ ).

The following conditions are equivalent.

(1) the cone $\operatorname{Ess}(X / Y)$ is of maximal dimension,

(2) the cone $\operatorname{Mov}(X / Y)$ is strictly convex, that is it contains no linear subspace of positive dimension,

(3) the classes of components of fibers of $\pi$ outside $\pi^{-1}(0)$ generate $N_{1}(X / Y)$,

(4) the classes of exceptional divisors generate $N^{1}(X / Y)$. 
Proof. In view of 3.5 the equivalence of (1) and (2) is formal. Also (1) is equivalent to (3) by the definition of the cone $\operatorname{Ess}(X / Y)$. Finally, the intersection of classes of exceptional divisors with curves contained in general fibers of their contraction is a non-degenerate pairing, c.f. 4.1. Hence (3) is equivalent to (4).

\section{Root systems and the structure of $\operatorname{Mov}(X / Y)$.}

4.1. Root systems. We recall some generalities regarding root systems: a standard reference for this part is Bou75. Consider a (finite dimensional) real vector space $V$ with a euclidean product, root lattice $\Lambda_{R}$ and weight lattice $\Lambda_{W}, \Lambda_{W} \supset \Lambda_{R}$. We distinguish the set of simple (positive) roots denoted by $\left\{e_{i}\right\}$ and their opposite $E_{i}=-e_{i}$. Note that the lattice $\Lambda_{R}$ is spanned by $E_{i}$ 's or $e_{i}$ 's while its $\mathbb{Z}$-dual is $\Lambda_{W}$. The Cartan matrix describes the intersection $\left(e_{i} \cdot e_{j}\right)=-\left(e_{i} \cdot E_{j}\right)$ which is also reflected in the respective Dynkin diagram. Any such root system is a (direct) sum of irreducible ones coming from the infinite series $\mathbb{A}_{n}, \mathbb{B}_{n}, \mathbb{C}_{n}, \mathbb{D}_{n}$ and also $\mathbb{E}_{6}, \mathbb{E}_{7}, \mathbb{E}_{8}$ as well as $\mathbb{F}_{4}$ and $\mathbb{G}_{2}$. By abuse we denote by the respective letter both the Cartan matrix and the associated root system.

The Cartan matrix of each of the systems $\mathbb{A}_{n}, \mathbb{D}_{n}$ and $\mathbb{E}_{6}, \mathbb{E}_{7}, \mathbb{E}_{8}$ has 2 at the diagonal and 0 or -1 outside the diagonal. Given a group $H$ of automorphisms of any of the $\mathbb{A}-\mathbb{D}-\mathbb{E}$ Dynkin diagrams we can produce a matrix of intersections of classes of orbits of the action. The entries are intersections of an element of the orbit with the sum of all elements in the orbit, that is: $\left(e_{i} \cdot \sum_{\left.e_{k} \in H_{(} e_{j}\right)} e_{k}\right)$. For example: the involution identifying two short legs of the $\mathbb{D}_{n}$ diagram $\cdots$ described by the $n \times n$ Cartan matrix

$$
\left(\begin{array}{rrrrr}
2 & 0 & -1 & 0 & \cdots \\
0 & 2 & -1 & 0 & \cdots \\
-1 & -1 & 2 & -1 & \cdots \\
0 & 0 & -1 & 2 & \cdots \\
\cdots & \cdots & \cdots & \cdots &
\end{array}\right)
$$

yields the $(n-1) \times(n-1)$ matrix associated with the system $\mathbb{C}_{n-1}$; we write $\mathbb{D}_{n} / \mathbb{Z}_{2}=\mathbb{C}_{n-1}$ :

$$
\left(\begin{array}{rrrr}
2 & -1 & 0 & \cdots \\
-2 & 2 & -1 & \cdots \\
0 & -1 & 2 & \cdots \\
\cdots & \cdots & \cdots &
\end{array}\right)
$$

Similarly, we verify that $\mathbb{A}_{2 n+1} / \mathbb{Z}_{2}=\mathbb{B}_{n}, \mathbb{D}_{n} / \mathbb{Z}_{2}=\mathbb{C}_{n-1}, \mathbb{E}_{6} / \mathbb{Z}_{2}=\mathbb{F}_{4}$ and $\mathbb{D}_{4} / \sigma_{3}=$ $\mathbb{G}_{2}$. The geometry behind these equalities is explained in Section 7.1

Let $\mathbb{U}_{n}$ denote the following $n \times n$ matrix

$$
\left(\begin{array}{rrrrr}
1 & -1 & 0 & 0 & \cdots \\
-1 & 2 & -1 & 0 & \cdots \\
0 & -1 & 2 & -1 & \cdots \\
0 & 0 & -1 & 2 & \cdots \\
\cdots & \cdots & \cdots & \cdots & \cdots
\end{array}\right)
$$

The matrix $\mathbb{U}_{n}$ is obtained from the root system $\mathbb{A}_{2 n}$ modulo involution of the respective Dynkin diagram. Here $\mathbb{U}$ stands for unreasonable (or un-necessary). 
4.2. The structure of Mov and Ess. The following is a combination of a result of Wierzba [Wie03, 1.3] and Theorem 3.5 .

Theorem 4.1. Let $\pi: X \rightarrow Y$ be a local symplectic contraction (arbitrary dimension). Suppose that $N^{1}(X / Y)$ is generated by the classes of codimension 1 components of the exceptional set of $\pi$, we call them $E_{\alpha}$; that is we are in situation described in Proposition 3.6. Let $e_{\alpha}$ denote the numerical equivalence class of an irreducible component of a general fiber of $\pi_{\mid E_{\alpha}}$. Then the following holds:

- The classes of $E_{\alpha}$ are linearly independent so they form a basis of $N^{1}(X / Y)$.

- The opposite of the intersection matrix $-\left(e_{\alpha} \cdot E_{\beta}\right)$ is a direct sum of Cartan matrices of type associated with simple algebraic Lie groups (or algebras), and possibly, matrices of type $\mathbb{U}_{n}$.

- If moreover $X$ is $M D S$ over $Y$ then $\operatorname{Mov}(X / Y)$ is dual, in terms of the intersection of $N^{1}(X / Y)$ and $N_{1}(X / Y)$, to the cone spanned by the classes of $e_{\alpha}$. In particular $\operatorname{Mov}(X / Y)$ is simplicial.

In short, the above theorem says that, apart from the case $\mathbb{U}_{n}$, which we do not expect to occur, the situation of an arbitrary local symplectic contraction on the level of divisors and 1-cycles is very much like in the case of the contraction to the nilpotent cone, which is the case of Theorem 7.4 and Corollary 7.5

Conjecture 4.2. The case $\mathbb{U}_{n}$ does not occur. That is, there is no symplectic contraction $X \rightarrow Y$ such that $Y$ has a codimension 2 locus of $\mathbb{A}_{2 n}$ singularities and there exists a non-trivial numerical equivalence for curves in $X$ which are in a general fiber of $\pi$ over this locus.

Since in dimension four $X$ is an MDS over $Y$, in order to prove this conjecture it is enough to deal with the case when $\pi: X \rightarrow Y$ is elementary and it is a contraction to $\mathbb{A}_{2}$ singularities in codimension 2 . Indeed, take an irreducible curve $C_{1}$ whose intersection with the irreducible divisor $E_{1}$ is $(-1)$; this is the upper-righthand corner of the matrix $\mathbb{U}_{n}$ in 4.1.1. The class of $C_{1}$ spans a ray on $\operatorname{Ess}(X / Y)$ and its dual $C_{1}^{\perp} \cap \operatorname{Mov}(X / Y)$ is a facet of $\operatorname{Mov}(X / Y)$. Hence we can choose an SQM model $X^{\prime}$ with a facet of $\operatorname{Nef}\left(X^{\prime} / Y\right)$ contained in $C_{1}^{\perp}$. Thus there exists an elementary contraction of $X^{\prime}$ which contracts $C_{1}$ whose exceptional locus is (the strict transform of) $E_{1}$.

Corollary 4.3. Suppose that the conjecture 4.2 is true. Then, for every local symplectic contraction $\pi: X \rightarrow Y$ satisfying the conditions of 3.6, there exists a semisimple Lie group and an identification of $N^{1}(X / Y)$ and $N_{1}(X / Y)$ with the real part of its Cartan algebra such that: (1) the intersection of the 1-cycles with classes of divisors is equal to the Killing form product, (2) the classes of irreducible essential curves spanning rays of $\operatorname{Ess}(X / Y)$ is identified with its primitive roots and (3) the cone $\operatorname{Mov}(X / Y)$ is identified with its Weyl chamber.

Conjecture 4.4. Under the above identification the classes of (integral) 1-cycles should form the lattive $\Lambda_{R}$ of roots, while the classes of divisors should make the lattice $\Lambda_{W}$ of weights.

4.3. Examples of root systems. We give a description of the cone of moving divisors and of the related root system for three examples coming as resolutions of $\mathbb{C}^{4} / G$, where $G<S p\left(\mathbb{C}^{4}\right)=: S p(4)$ is a finite subgroup preserving a symplectic form. The reader unfamiliar with these examples may prefer to read first Section 6. 
Example 4.5. Let $B T$ be the binary tetrahedral group; that is $B T$ is the preimage under the standard homomorphism $S U(2) \rightarrow S O(3)$ of the subgroup of $S O(3)$ generated by the symmetries of a regular tetrahedron. This group has three irreducible representations on $\mathbb{C}^{2}$, the standard arising from the embedding into $S U(2)$ and two other one called $S_{1}$ and $S_{2}$. The group $B T$ acts on the product $S_{1} \otimes S_{2}=\mathbb{C}^{4}$. It is known that $\mathbb{C}^{4} / B T$ has a symplectic resolution; in fact, recently Lehn and Sorger gave an explicit construction of it, see [S08. This resolution is related to the root system $\mathbb{A}_{2}$ with generators $e_{1}$ and $e_{2}$. Then $v= \pm\left(e_{1}-e_{2}\right)$ is the flopping system. Here is the picture of the weight lattice, together with roots denoted by - and flopping classes denoted by o. The Mov cone (or Weyl chamber) is divided into two parts by the line orthogonal to the flopping class.

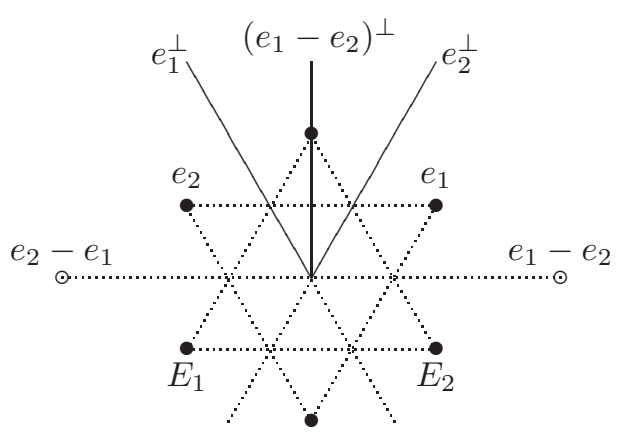

Example 4.6. Take the quotient $\mathbb{C}^{4} / \mathbb{Z}_{2}^{2} \rtimes \mathbb{Z}_{2}$ and its Hilb-Chow resolution $X \rightarrow Y$; this is Example 2.4 for $n=2$, more details can be found in Section 6. It is related to the decomposable root system $\mathbb{A}_{1} \oplus \mathbb{A}_{1}$ with roots denoted, respectively, by $e_{0}$ and $e_{1}$. The following picture describes a section of Mov together with its decomposition by flopping classes.

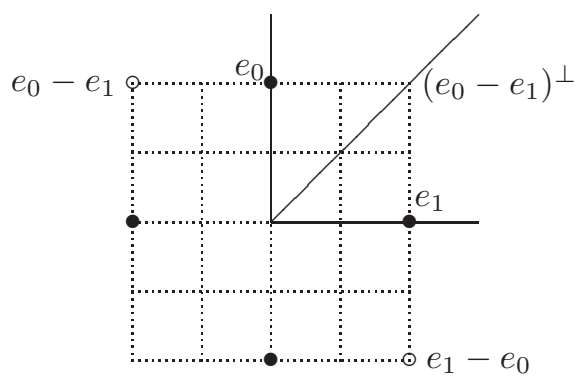

Example 4.7. Take the quotient $Y=\mathbb{C}^{4} / \mathbb{Z}_{3}^{2} \rtimes \mathbb{Z}_{2}$ and its Hilb-Chow resolution $X \rightarrow Y$; this is Example 2.4 for $n=3$. It is related to the decomposable root system $\mathbb{A}_{2} \oplus \mathbb{A}_{1}$ with roots denoted, respectively, by $e_{1}, e_{2}$ and $e_{0}$. The following picture describes a plane section of a 3 -dimensional cone $\operatorname{Mov}(X / Y)$ (denoted by solid line segments) together with its decomposition by flopping classes (denoted by dotted line segments). The upper chamber in this picture is the nef cone $\operatorname{Nef}(X / Y)$. This 
situation will be discussed in detail in 6.5 and 6.6 .

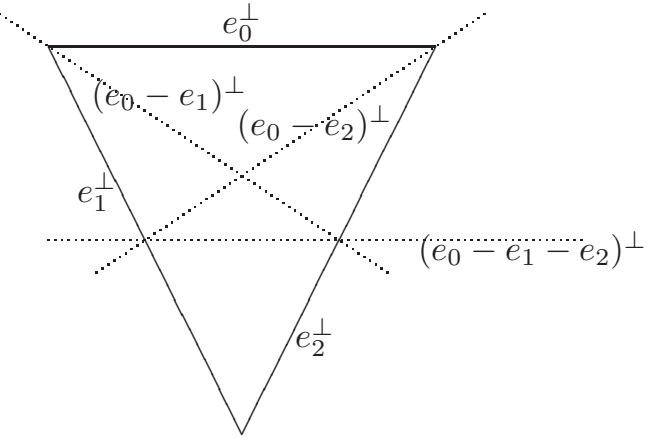

\section{Rational CURVES AND DifFerential FORMS}

5.1. The set-up. Let $\pi: X \rightarrow Y$ be a local symplectic contraction of a 4-fold. We assume that we are in the situation of 3.6. In particular, the exceptional locus of $\pi$ is a divisor $D$. This divisor, as well as its image surface $S:=\pi(D) \subset Y$, can be reducible. As above $0 \in S \subset Y$ denotes the unique point over which $\pi$ can have a two dimensional fiber.

Our starting point is the paper of Wierzba Wie03 (as well as the appendix of [SCW04]) to which we will often refer. In particular Theorem 1.3 of Wie03 says that a general fiber of $\pi$ over any component of $S$ is a configuration of $\mathbb{P}^{1}$ 's with dual graph being a Dynkin diagram. The components of these fibers are called essential curves in the previous section.

Choose an irreducible component of $S$, call it $S^{\prime}$. Take an irreducible curve $C \simeq \mathbb{P}^{1}$ in a (general) fiber over a point in $S^{\prime} \backslash\{0\}$ and let $D^{\prime}$ be the irreducible component of $D$ which contains $C$; note that $\pi\left(D^{\prime}\right)=S^{\prime}$ and $S^{\prime}$ may be (and usually is) nonnormal. Let $\mathcal{V}^{\prime} \subset \operatorname{Chow}(X / Y)$ be an irreducible component of the Chow scheme of $X$ containing $C$. By $\mathcal{V}$ we denote its normalization and $p: \mathcal{U} \rightarrow \mathcal{V}$ is the normalized pullback of the universal family over $\mathcal{V}^{\prime}$. Finally, let $q: \mathcal{U} \rightarrow D^{\prime} \subset X$ be the evaluation map, see e.g. Kol96, I.3] for the construction. The contraction $\pi$ determines a morphism $\tilde{\pi}: \mathcal{V} \rightarrow S^{\prime}$, which is surjective because $C$ was chosen in a general fiber over $S^{\prime}$. We let $\mu: \mathcal{V} \rightarrow \widetilde{S}^{\prime} \rightarrow S^{\prime}$ be its Stein factorization. In particular $\widetilde{S}^{\prime}$ is normal and $\nu: \widetilde{S}^{\prime} \rightarrow S^{\prime}$ is a finite morphism, ètale outside $\nu^{-1}(0)$, whose fibers are related to the orbits of the action of the group of automorphism of the Dynkin diagram, Wie03, 1.3]. We will assume that $\mu$ is not an isomorphism which is equivalent to say that $D^{\prime}$ has a 2-dimensional fiber over 0 . Also, since we are interested in understanding the local description of the contraction in analytic category we will assume that $S^{\prime}$ is analytically irreducible at 0 or that $\nu^{-1}(0)$ consists of single point. The exceptional locus of $\mu$ is $\mu^{-1}\left(\nu^{-1}(0)\right)=\bigcup_{i} V_{i}$ where $V_{i} \subset \mathcal{V}$ are irreducible curves.

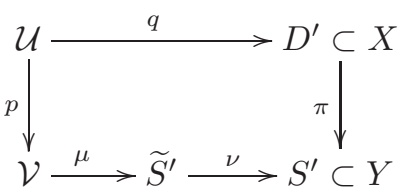


If necessary, we can take $\mathcal{V}$ to be smooth, eventually by replacing it with its desingularization and $\mathcal{U}$ with the normalized fiber product. A general fiber of $p: \mathcal{U} \rightarrow \mathcal{V}$ is $\mathbb{P}^{1}$ while other fibers are, possibly, trees of rational curves. If $C$ is an extremal curve, which by 3.5 and 3.2 is true for some SQM model of $X$, then $-D$ is ample on the extremal ray spanned by $C$ and since $-D \cdot C \leq 2$ it follows that $p: \mathcal{U} \rightarrow \mathcal{V}$ is a $\mathbb{P}^{1}$ or conic bundle. Since any two SQM models of $X$ are obtained by a sequence of Mukai flops, it follows that in a general situation $p: \mathcal{U} \rightarrow \mathcal{V}$ is obtained by a sequence of blows and blow-downs of a $\mathbb{P}^{1}$ or conic bundle.

In Wie03 and [SCW04 it was proved that $S^{\prime} \backslash\{0\}$ is smooth and that, on $\mathcal{V} \backslash\{(\nu \circ$ $\left.\mu)^{-1}(0)\right\}, p$ is a $\mathbb{P}^{1}$-bundle. It was also showed, by pulling back the symplectic form via $q$ and pushing it further down via $p$, that one can obtain a symplectic form on $S^{\prime} \backslash\{0\}$. We will repeat their procedure in this more general case.

5.2. The differentials. Let us consider the derivative map $D q: q^{*} \Omega_{X} \rightarrow \Omega_{\mathcal{U}}$. Its cokernel is a torsion sheaf, call it $\mathcal{Q}_{\Delta_{2}}$, supported on the set $\Delta_{2}$, which is the set of points where $q$ is not of maximal rank: by the theorem on the purity of the branch locus $\Delta_{2}$ is a divisor. As for the kernel, let $I$ be the ideal of $D^{\prime}$ in $X$ and consider the sequence $q^{*}\left(I / I^{2}\right) \rightarrow q^{*} \Omega_{X} \rightarrow \Omega_{\mathcal{U}}$. The saturation of the image of the first map will be the kernel of the second map and it will be a reflexive sheaf of the form $\mathcal{O}_{\mathcal{U}}\left(-D^{\prime}+\Delta_{1}\right)$, with $\Delta_{1}$ being an effective divisor. In the above notation we can write the exact sequence

$$
0 \longrightarrow \mathcal{O}_{\mathcal{U}}\left(q^{*}\left(-D^{\prime}\right)+\Delta_{1}\right) \longrightarrow q^{*} \Omega_{X} \longrightarrow \Omega_{\mathcal{U}} \longrightarrow \mathcal{Q}_{\Delta_{2}} \longrightarrow 0 .
$$

We have another derivation map into $\Omega_{\mathcal{U}}$, namely $D p: p^{*} \Omega_{\mathcal{V}} \rightarrow \Omega_{\mathcal{U}}$. It fits in the exact sequence

$$
p^{*} \Omega_{\mathcal{V}} \longrightarrow \Omega_{\mathcal{U}} \longrightarrow \Omega_{\mathcal{U} / \mathcal{V}} \longrightarrow 0,
$$

whose dual sequence is

$$
0 \longrightarrow T_{\mathcal{U} / \mathcal{V}} \longrightarrow T_{\mathcal{U}} \longrightarrow p^{*} T_{\mathcal{V}}
$$

The symplectic form on $X$, that is $\omega_{X}$, gives an isomorphism $\omega_{X}: T_{X} \rightarrow \Omega_{X}$. We consider the following diagram involving morphism of sheaves over $\mathcal{U}$ appearing in the above sequences.

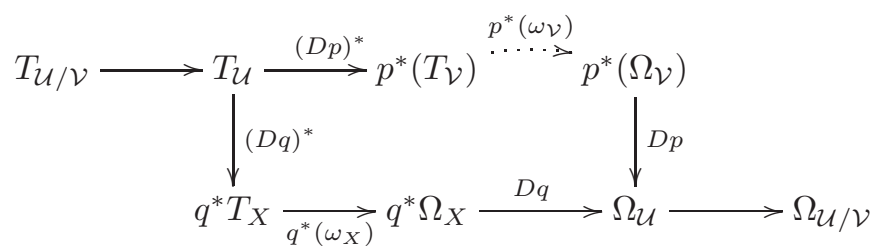

We claim that the dotted arrow exists and it is obtained by a pull back of a two form $\omega_{\mathcal{V}}$ on $\mathcal{V}$, and it is an isomorphism outside the exceptional set of $\mu$ which is $\bigcup_{i} V_{i}$. Indeed, the composition of arrows in the diagram which yields $T_{\mathcal{U}} \rightarrow \Omega_{\mathcal{U}}$ is given by the 2 -form $D q\left(\omega_{X}\right)$ and it is zero on $T_{\mathcal{U} / \mathcal{V}}$, because this is a torsion free sheaf and its restriction to any fiber of $p$ outside $\bigcup_{i} V_{i}$ (any fiber of $p$ is there a 
$\left.\mathbb{P}^{1}\right)$ is $\mathcal{O}(2)$ while the restriction of $\Omega_{\mathcal{U}}$ is $\mathcal{O}(-2) \oplus \mathcal{O} \oplus \mathcal{O}$. By the same reason the composition $T_{\mathcal{U}} \rightarrow \Omega_{\mathcal{U}} \rightarrow \Omega_{\mathcal{U} / \mathcal{V}}$ is zero since $T_{\mathcal{U}}$ on any fiber of $p$ outside $\bigcup_{i} V_{i}$ is $\mathcal{O}(2) \oplus \mathcal{O} \oplus \mathcal{O}$ while $\Omega_{\mathcal{U} / \mathcal{V}}$ is $\mathcal{O}(-2)$. Thus the map $D q\left(\omega_{X}\right): T_{\mathcal{U}} \rightarrow \Omega_{\mathcal{U}}$ factors through $p^{*}\left(T_{\mathcal{V}}\right) \rightarrow p^{*}\left(\Omega_{\mathcal{V}}\right)$ and, as a result, $D q\left(\omega_{X}\right)=D p\left(\omega_{\mathcal{V}}\right)$, for some 2 -form $\omega_{\mathcal{V}}$ on $\mathcal{V}$. Since $D q$ is of maximal rank outside of $p^{-1}\left(\bigcup_{i} V_{i}\right)$ and $p$ is just a $\mathbb{P}^{1}$-bundle there, it follows that $\omega_{\mathcal{V}}$ does not assume zero outside the exceptional set of $\mu$. Hence $K_{\mathcal{V}}=\sum a_{i} V_{i}$, with $a_{i} \geq 0$ being the discrepancy of $V_{i}$. Note that the above argument follows essentially from [SCW04, Sect. 4.1] or [Wie03, Sect. 5].

Theorem 5.1. The surface $\widetilde{S}^{\prime}$ has at most Du Val (or $\mathbb{A}-\mathbb{D}-\mathbb{E}$ ) singularity at $\nu^{-1}(0)$ and $\mu: \mathcal{V} \rightarrow \widetilde{S}^{\prime}$ is its, possibly non-minimal, resolution. In particular every $V_{i}$ is a rational curve. If a component $V_{i}$ has positive discrepancy or, equivalently, the form $\omega_{\mathcal{V}}$ vanishes along $V_{i}$, then $p^{-1}\left(V_{i}\right) \subset \Delta_{2}$.

Proof. The first statement follows from the discussion preceeding the theorem. To get the second part, note that over $\mathcal{U}$ we have $D q\left(\omega_{X}\right)=D p\left(\omega_{\mathcal{V}}\right)$ and $\omega_{\mathcal{V}}$ is zero at any component of $\bigcup_{i} V_{i}$ of positive discrepancy. Since $\omega_{X}$ is nondegenerate this equality implies that $D q$ is of rank $\leq 2$ on the respective component of $p^{-1}\left(\bigcup V_{i}\right)$.

We note that although the surface $\widetilde{S}^{\prime}$ is the same for all the symplectic resolutions of $Y$, the parametric scheme for lines, which is a resolution of $\widetilde{S}^{\prime}$ may be different for different SQM models, see 6.5 for an explicit example.

Proposition 5.2. Suppose that the map $p$ is of maximal rank in codimension 1. Then the p-inverse image of the set of positive discrepancy components of $\bigcup_{i} V_{i}$ coincides with the set where the rank of $q$ drops. That is, $\Delta_{2}$ is the pullback of the zero set of $\omega_{\mathcal{V}}$.

Proof. We have the following injective morphism of sheaves $q^{*}\left(\omega_{X}\right) \circ(D q)^{*}\left(T_{\mathcal{U} / \mathcal{V}}\right) \hookrightarrow$ $\mathcal{O}_{\mathcal{U}}\left(-p^{*} D+\Delta_{1}\right) \hookrightarrow q^{*} \Omega_{X}$ which follows, as already noted, because of the splitting type of $\Omega_{\mathcal{U}}$. We claim that this implies the isomorphism of line bundles $T_{\mathcal{U} / \mathcal{V}} \simeq \mathcal{O}_{\mathcal{U}}\left(-p^{*} D+\Delta_{1}\right)$. Indeed, the evaluation map of the universal family over the Chow scheme is isomorphic on the fibers, hence $(D q)^{*}$ is of maximal rank along $T_{\mathcal{U} / \mathcal{V}}$ is codimension 1 at least, hence the desired isomorphism.

Now, since $p$ is submersive in codimension 1 , because of the sequence 5.2 .7 we can write $\operatorname{det} \Omega_{\mathcal{U}}=p^{*}\left(K_{\mathcal{V}}\right) \otimes \Omega_{\mathcal{U} / \mathcal{V}}$ and consequently, because of the sequence [5.2.6. we get

$c_{1}\left(\mathcal{Q}_{\Delta_{2}}\right)=c_{1}\left(\mathcal{O}_{\mathcal{U}}\left(-p^{*} D+\Delta_{1}\right)\right)-c_{1}\left(T_{\mathcal{U} / \mathcal{V}}\right)+c_{1}\left(p^{*}\left(K_{\mathcal{V}}\right)\right)=c_{1}\left(p^{*} K_{\mathcal{V}}\right)=p^{*}\left(\sum a_{i}\left[V_{i}\right]\right)$

5.3. Vertical slicing. The first of the following two results is essentially known, c.f. Kal06, 2.3] and also [Wie03, 1.2(ii), 1.4.]. We restate and reprove it in the form suitable for the subsequent corollary.

Proposition 5.3. Suppose that $\pi: X \rightarrow Y$ is a symplectic contraction with $\operatorname{dim} X=2 n$. Let $Z \subset X$, with $\operatorname{codim} Z=m$, be a (irreducible) maximal cycle with $S=\pi(Z)$, codim $S=2 m$. The fibers of $\pi_{\mid Z}: Z \rightarrow S$ are isotropic (with respect to $\omega_{X}$ ) and, moreover, over an open and dense set $S_{0} \subset S$ there exists a symplectic form $\omega_{S}$ such that over $\pi_{\mid Z}^{-1}\left(S_{0}\right)$ we have $D \pi\left(\omega_{S}\right)=\omega_{X \mid Z}$ 
Proof. The proof that $\omega_{X}$ restricted to fibers of $\pi$ is zero, so that they are isotropic (or lagrangian), is in WW03, 2.20]. Let $\iota: Z \rightarrow X$ be the embedding. Then we have the following version of diagram 5.2 .9

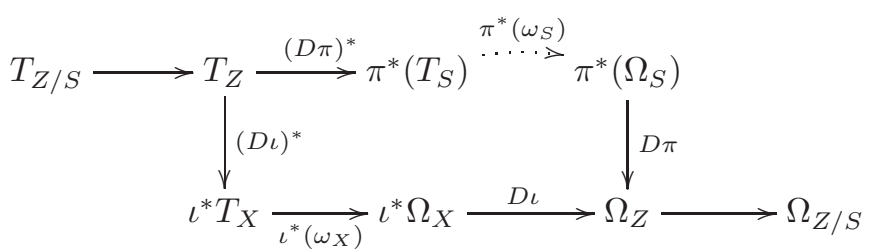

We claim the existence of $\omega_{S}$. The composition $T_{Z / S} \rightarrow \Omega_{Z / S}$ is trivial since fibers of $\pi$ are isotropic. On the other hand the induced maps $\pi^{*}\left(T_{S}\right) \rightarrow \Omega_{Z / S}$ and $T_{Z / S} \rightarrow \pi^{*} \Omega_{S}$ are zero: indeed, otherwise we would have nonzero 1 forms on a generic fiber of $\pi_{\mid Z}$, which would contribute to the first cohomology of the fiber (via the Hodge theory on the simplicial resolution of the fiber) which contradicts Kal06, 2.12].

Thus the dotted arrow in the above diagram is well defined and it satisfies $D \pi\left(\omega_{S}\right)=$ $D \iota\left(\omega_{X}\right)$ for a two form $\omega_{S}$ defined over a smooth subset $S_{0}$ of $S$. Moreover the form $\omega_{S}$ is of maximal rank for the dimensional reasons.

The following corollary is a symplectic version of [AnWi98, 1.3].

Corollary 5.4. [Vertical slicing] In the situation of 5.3 let $H_{1}, \ldots H_{2 n-2 m}$ be general irreducible divisors in $Y$ meeting in a general point $s \in S$. Letting $Y^{\prime}=$ $H_{1} \cap \cdots \cap H_{2 n-2 m}$ and $X^{\prime}=\pi^{-1}\left(Y^{\prime}\right)$, possibly shrinking $Y^{\prime}$ to a neighborhood of $s$, we get that $\pi^{\prime}=\pi_{\mid X^{\prime}}: X^{\prime} \rightarrow Y^{\prime}$ is a local symplectic contraction of a $2 m$-fold with an exceptional fiber, $\pi^{-1}(s)$, of dimension $m$.

Proof. Since $\pi$ is crepant it is enough to show that the restriction of $\omega_{X}$ to $X^{\prime}$ is nondegenerate at a point over $s$ in order to claim that it is symplectic over the whole $X^{\prime}$ (after possibly shrinking $Y^{\prime}$ to a neighborhood of $s$ ). To this end we consider the following commuting diagram with exact rows

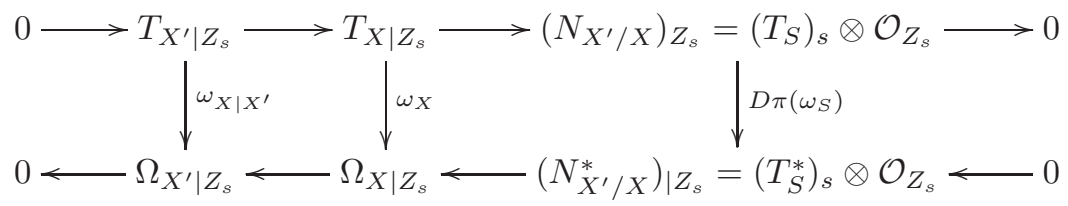

Here $Z_{s}=Z \cap X^{\prime}$ is a complete intersection, hence $\left(N_{X^{\prime} / X}\right)_{\mid Z_{s}}=N_{Z_{s} / Z}$ which yields the identifications in the last non-zero column of the diagram. The righthand-side vertical arrow follows because of 5.3 , where we have also shown that is an isomorphism. This implies that the left-hand-side vertical arrow is an isomorphism too.

\section{Quotient Symplectic Singularities}

6.1. Preliminaries. In this section $G<S p\left(\mathbb{C}^{4}\right)=: S p(4)$ is a finite subgroup preserving a symplectic form. We will discuss some examples in which $Y:=\mathbb{C}^{4} / G$ admits a symplectic resolution $\pi: X \rightarrow Y$. We have the following two fundamental results about such resolutions, the latter one known as the McKay correspondence. 
Theorem 6.1. (c.f. Ver00) If $Y$ admits a symplectic resolution then $G$ is generated by symplectic reflections, that is elements whose fixed points set is of codimension 2.

Theorem 6.2. (c.f. Kal02]) The homology classes of the maximal cycles (as defined in 2.2) form a basis of rational homology of $X$ and they are in bijection with conjugacy classes of elements of $G$.

On the other hand we have the following immediate observation (for further details see for instance section 3.2 in [AnWi10]).

Lemma 6.3. Let $S^{\prime} \subset Y$ be a component of the codimension 2 singular locus associated with the isotropy group $H<G$. Then $H$ is one of the $\mathbb{A}-\mathbb{D}-\mathbb{E}$ groups (a finite subgroup of $S L\left(\mathbb{C}^{2}\right)$ ) consisting of symplectic reflections. The normalization of $S^{\prime}$ has a quotient singularity by the action of $W(H)=N_{G}(H) / H$, where $N_{G}(H)$ is the normalizer of $H$ in $G$.

6.2. Direct product resolution. Let $H_{1}, H_{2}<S L(2)$ be finite subgroups and consider $G:=H_{1} \times H_{2}$ acting on $\mathbb{C}^{4}=\mathbb{C}^{2} \times \mathbb{C}^{2}$. Let $\pi_{i}: S_{i} \rightarrow \mathbb{C}^{2} / H_{i}$ be minimal resolutions and $n_{i}=\left|H_{i}\right|-1$ be the number of exceptional rational curves in $S_{i}$. The product morphism $\pi=\pi_{1} \times \pi_{2}: X:=S_{1} \times S_{2} \rightarrow Y:=\mathbb{C}^{4} / G$ is a symplectic resolution with the central fiber isomorphic to the product of the exceptional loci of $\pi_{i}$. In particular $X$ does not admit any flop and $\operatorname{Mov}(X / Y)=\operatorname{Nef}(X / Y)$. Every component of $\operatorname{Chow}(X / Y)$ containing an exceptional curve of $\pi_{i}$ is isomorphic to $S_{j}$, with $i \neq j \in\{1,2\}$.

6.3. Elementary contraction to $\mathbb{C}^{4} / \sigma_{3}$. Let $\sigma_{3}$ be a group of permutation of 3 elements; $\sigma_{3}$ acts on $\mathbb{C}^{2}$ via the standard representation. Let $\sigma_{3}$ acts on $\mathbb{C}^{4}=\mathbb{C}^{2} \oplus$ $\mathbb{C}^{2}$ as the diagonal action of the standard representation. A symplectic resolution of the quotient $\mathbb{C}^{4} / \sigma_{3}$ can be obtained as a section of the Hilbert-Chow morphism $\tau: H i l b^{3}\left(\mathbb{C}^{2}\right) \rightarrow\left(\mathbb{C}^{2}\right)^{(3)}$ in 2.4. This is a local version of Beauville's construction, Bea83 (see also AnWi10), and it can be seen as a special case of 5.4. More explicit calculations on this resolution are done in section 7.2 .

There are three conjugacy classes in $\sigma_{3}$ which are related to three maximal cycles, of complex dimension 4, 3 and 2, each related to a 1-dimensional group of homology for the resolution $\pi: X \rightarrow Y=\mathbb{C}^{4} / \sigma_{3}$.

Since the normalizer of any order 2 element in $\sigma_{3}$ (any transposition or any reflection, if one thinks about $\sigma_{3}$ as the dihedral group) is trivial, by Lemma 6.3 it follows that the normalization of the singular locus $S$ of $Y$ is smooth. Hence, by 5.2 we can compute both the parametrizing scheme for rational curves in $X$ and the respective universal family. That is, the parametrizing scheme $\mathcal{V}$ is just a blow-up of the normalization of $S$, the evaluation map $q: \mathcal{U} \rightarrow X$ drops its rank over 0 and the exceptional divisor of $\pi$, which is the image of $q$, is non-normal over 0 .

6.4. Wreath product. Let $H<S L(2)$ be a finite subgroup and let $G:=H^{\times 2} \rtimes \mathbb{Z}_{2}$ where $\mathbb{Z}_{2}$ interchanges the factors in the product. We write $G=H \imath \mathbb{Z}_{2}$. Note that $\mathbb{Z}_{n+1} \prec \mathbb{Z}_{2}$ has another nice presentation, namely $\left(\mathbb{Z}_{n+1}\right)^{\times 2} \rtimes \mathbb{Z}_{2}=D_{2 n} \rtimes \mathbb{Z}_{n}$, where $D_{2 n}$ is the dihedral group of the regular $n$-gon and $\mathbb{Z}_{n}$ acts on it by rotations.

We consider the projective symplectic resolution described in 2.4 (with $n=2$ ):

$$
\pi: X:=\operatorname{Hilb}^{2}(S) \rightarrow S^{(2)} \rightarrow\left(\mathbb{C}^{2} / H\right)^{(2)}:=Y
$$


where $\nu: S \rightarrow \mathbb{C}^{2} / H$ is the minimal resolution with the exceptional set $\bigcup_{i} C_{i}$, where $C_{i}, i=1, \ldots, k$, are $(-2)$-curves.

The morphism $\tau: \operatorname{Hilb}^{2}(S) \rightarrow S^{(2)}$ is just a blow-up of the locus of $\mathbb{A}_{1}$ singularities (the image of the diagonal under $S^{2} \rightarrow S^{(2)}$ ) with irreducible exceptional divisor $E_{0}$ which is a $\mathbb{P}^{1}$ bundle over $S$. We set $S^{\prime}=\pi\left(E_{0}\right)$. By $E_{i}$, with $i=1, \ldots, k$ we denote the strict transform, via $\tau$, of the image of $C_{i} \times S$ under the map $S^{2} \rightarrow S^{(2)}$. By $e_{i}$ we denote the class of an irreducible component of a general fiber of $\pi_{\mid E_{i}}$. The image $\pi\left(E_{i}\right)$ for $i \geq 1$ is the surface $S^{\prime \prime} \simeq C^{2} / H$. The singular locus of $Y$ is the union $S=S^{\prime} \cup S^{\prime \prime}$.

The irreducible components of $\pi^{-1}(0)$ are described in the following.

- $P_{i, i}$, for $i=1, \ldots, k$. They are the strict transform of $C_{i}^{(2)}$ via $\tau$. They are isomorphic to $\mathbb{P}^{2}$.

- $P_{i, j}$, for $i, j=1, \ldots, k$ and $i<j$. They are the strict transform via $\tau$ of the image of $C_{i} \times C_{j}$ under the morphism $S^{2} \rightarrow S^{(2)}$. They are isomorphic to $\mathbb{P}^{1} \times \mathbb{P}^{1}$ if $C_{i} \cap C_{j}=\emptyset$ and to the blow up of $\mathbb{P}^{1} \times \mathbb{P}^{1}$ if $C_{i} \cap C_{j} \neq \emptyset$.

- $Q_{i}$, for $i=1, \ldots, k$. They are the preimage $\tau^{-1}\left(\Delta_{C_{i}}\right)$, where $\Delta_{C_{i}}$ is the diagonal embedding of $C_{i}$ in $S^{(2)}$. They are isomorphic to $\mathbb{P}\left(T_{S \mid C_{i}}\right)=$ $\mathbb{P}\left(\mathcal{O}_{C_{i}}(2) \oplus \mathcal{O}_{C_{i}}(-2)\right)$, i.e. to the Hirzebruch surface $F_{4}$.

Let us also describe some intersections between these components. Namely, $P_{i, i}$ intersects $Q_{i}$ along a curve which is a $(-4)$-curve in $Q_{i}$ and a conic in $P_{i, i}$. If $C_{i} \cap C_{j}=\left\{x_{i}\right\}$ then $P_{i, j}$ intersect $P_{i, i}$ (respectively $P_{j, j}$ ) along a curve which is a $(-1)$ curve in $P_{i, j}$ and a line in $P_{i, i}$ (respectively in $P_{j, j}$ ). Moreover in this case $P_{i, j}$ intersect $Q_{i}$ (respectively $Q_{j}$ ) in a curve which is a $(-1)$ curve in $P_{i, j}$ and a fiber in $Q_{i}$ (respectively $Q_{j}$ ).

The next lemma is straightforward, a proof of it can be found in Fu06b, Lemma 4.2].

Lemma 6.4. The strict transform of $Q_{i}$ under any sequence of Mukai flops along components in $\pi^{-1}(0)$ is not isomorphic to $\mathbb{P}^{2}$.

6.5. Resolutions of $\mathbb{C}^{4} /\left(\mathbb{Z}_{3} \backslash \mathbb{Z}_{2}\right)$. The Figure 1 presents a "realistic" description of configurations of components in the special fiber of symplectic resolutions of $\mathbb{C}^{4} /\left(\mathbb{Z}_{3} \backslash \mathbb{Z}_{2}\right)$. By abuse, the strict transforms of the components and the results of the flopping of $\mathbb{P}^{2}$ 's are denoted by the same letters.

The position of these configurations in Figure 1 is consistent with the decomposition of the cone $\operatorname{Mov}(X / Y)$ presented in Diagram 4.3.4. In particular, the configuration at the top is associated with the Hilbert-Chow resolution. Note that the central configuration of this diagram cointains three copies of $\mathbb{P}^{2}$, denoted $P_{i j}$, which contain lines whose classes are $e_{0}-e_{1}, e_{0}-e_{2}$ and $e_{1}+e_{2}-e_{0}$.

On the other hand, the configuration in the bottom is associated with the resolution which can be factored by two different divisorial elementary contractions of classes $e_{1}$ and $e_{2}$. In fact, contracting both $e_{1}$ and $e_{2}$ is a resolution of $\mathbb{A}_{2}$ singularities which is a part of a resolution of $Y$ which comes from presenting $\mathbb{Z}_{3} \prec \mathbb{Z}_{2}$ as $D_{6} \rtimes \mathbb{Z}_{3}$. That is, $X$ is then obtained by first resolving the singularities of the action of $D_{6}=\sigma_{3}$ and then by resolving the singularities of the $\mathbb{Z}_{3}$ action on this resolution. The rulings of the respective surfaces coming from this last blow up are indicated by dotted line segments. We will call such $X$ a $D_{6} \rtimes \mathbb{Z}_{3}$-resolution.

This example is convenient for understanding the contents of Theorem 5.1 and of Proposition 5.2 We refer to diagram 5.1.5 and let $S^{\prime}$ and $S^{\prime \prime}$ be the closure of the 

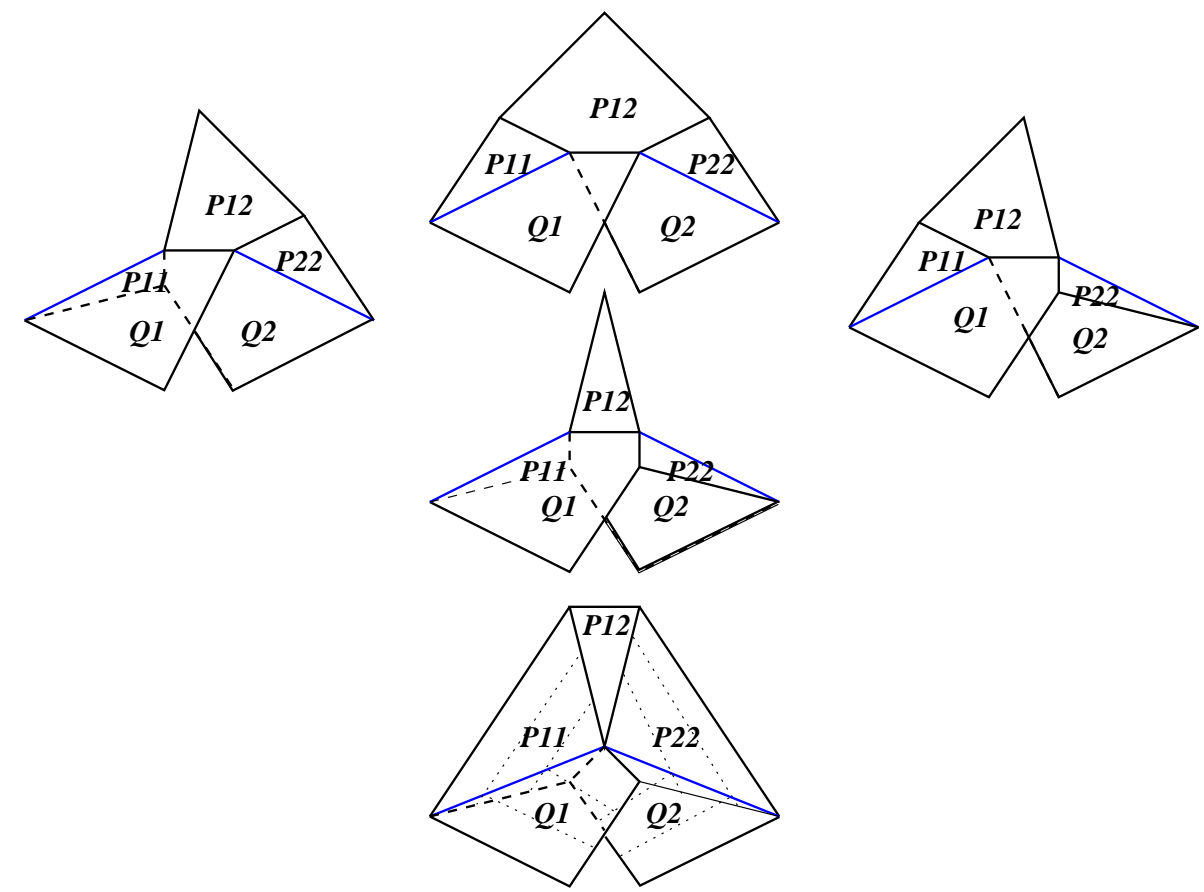

Figure 1. Components of the central fiber in resolutions of $\mathbb{C}^{4} /\left(\mathbb{Z}_{3} \succ \mathbb{Z}_{2}\right)$

locus of $\mathbb{A}_{1}$ and $\mathbb{A}_{2}$ singularities in $Y=\mathbb{C}^{4} /\left(\mathbb{Z}_{3} \prec \mathbb{Z}_{2}\right)$. From Lemma 6.3 we find out that the normalization of $S^{\prime}$ as well as $S^{\prime \prime}$ has a singularity of type $\mathbb{A}_{2}$.

By $\mathcal{V}_{0}$ we denote the component of $\operatorname{Chow}(X / Y)$ dominating $S^{\prime}$ and parametrizing curves equivalent to $e_{0}$, while by $\mathcal{V}_{1}$ and $\mathcal{V}_{2}$ we denote components dominating $S^{\prime \prime}$ parameterizing deformations of $e_{1}$ and $e_{2}$. The surfaces $\mathcal{V}_{i}$ may depend on the resolution and, in fact, while $\mathcal{V}_{1}$ and $\mathcal{V}_{2}$ remain unchanged, the component $\mathcal{V}_{0}$ will change under flops.

Lemma 6.5. If $X$ is the Hilbert-Chow resolution then $\mathcal{V}_{0}$ is the minimal resolution of $\mathbb{A}_{2}$ singularity. If $X$ is the $D_{6} \rtimes \mathbb{Z}_{3}$-resolution then $\mathcal{V}_{0}$ is non-minimal, with one $(-1)$ curve in the central position of three exceptional curves.

Proof. The first statement is immediate. To see the second one, note that we have the map of $\mathcal{V}_{0}$ to Chow of lines in the resolution of $\mathbb{C}^{4} / \sigma_{3}$ divided by $\mathbb{Z}_{3}$ action. The $\mathbb{Z}_{3}$-action in question is just a lift up of the original linear action on the fixed point set of rotations in $\sigma_{3}=D_{6}$ hence $\mathcal{V}_{0}$ resolves 2 cubic cone singularities associated with the eigenvectors of the original action.

One may verify that in the $D_{6} \rtimes \mathbb{Z}_{3}$-resolution the exceptional set in $\mathcal{V}_{0}$ parametrizes curves consisting of three components: $\mathbb{Q}_{2} \cap P_{11}, Q_{1} \cap P_{22}$ and a line in $P_{12}$, whose classes are, respectively, $e_{2}, e_{1}$ and $e_{0}-\left(e_{1}+e_{2}\right)$.

6.6. Resolutions of $\mathbb{C}^{4} /\left(\mathbb{Z}_{n+1} \prec \mathbb{Z}_{2}\right)$. We use the notation introduced in Theorem 4.1. Corollary 4.3 and Theorem 3.3 . Suppose to be in the set up of 6.4 with 
$H=\mathbb{Z}_{n+1}$. In particular the classes $e_{i}$, for $i=1, \ldots, n$, are identified to simple roots associated with consecutive nodes of the Dynkin diagram $\mathbb{A}_{n}$.

Theorem 6.6. Let $X \rightarrow Y=\mathbb{C}^{4} /\left(\mathbb{Z}_{n+1} \prec \mathbb{Z}_{2}\right)$ be a symplectic resolution as above. The division of $\operatorname{Mov}(X / Y)=\left\langle e_{0}, \ldots, e_{n}\right\rangle^{\vee}$ into Mori chambers is defined by hyperplanes $\lambda_{i j}^{\perp}$ for $1 \leq i \leq j \leq n$, where $\lambda_{i j}=e_{0}-\left(e_{i}+e_{i+1}+\cdots+e_{j-1}+e_{j}\right)$.

A proof of this theorem will occupy the rest of this section.

We know one Mori chamber of $\operatorname{Mov}(X / Y)$, the one associated with the HilbertChow resolution. The faces of this chamber are supported by $e_{0}^{\perp}$ and by $-\lambda_{i i}^{\perp}=$ $\left(e_{i}-e_{0}\right)^{\perp}$, see e.g. the above discussion. Thus, in particular, if $\lambda \in N_{1}(X)$ is a flopping class then $\lambda^{\perp}$ does not meet the relative interior of the face $\operatorname{Mov}(X) \cap e_{0}^{\perp}$. On the other hand, $\operatorname{Mov}(X / Y)=\operatorname{Mov}(X / Y) \cap e_{0}^{\perp}+\mathbb{R}_{\geq 0} \cdot\left(-E_{0}\right)$. Thus, if we take any $D_{0}$ in the relative interior of $\operatorname{Mov}(X) \cap e_{0}^{\perp}$ then, by the above observation, the half-line $D_{0}+\mathbb{R}_{\geq 0} \cdot\left(-E_{0}\right)$ must meet the hyperplane $\lambda^{\perp}$, for any flopping class $\lambda$. Hence the theorem will be proved if, for a choice of $D_{0}$, we will show that all the hyperplanes $\lambda^{\perp}$ that $D_{0}+\mathbb{R}_{\geq 0} \cdot\left(-E_{0}\right)$ meets actually come from the classes $\lambda_{i j}$.

Let us choose a sequence (a vector) of $n$ positive numbers $\bar{\beta}=\left(\beta_{i}\right)$ such that $\beta_{1}+\cdots+\beta_{i-1}<\beta_{i}$, for $i=2, \ldots, n$. We set $\gamma_{i j}=\beta_{i}+\cdots+\beta_{j}$. Then, by our assumption,

$$
\gamma_{11}<\gamma_{22}<\gamma_{12}<\gamma_{33}<\gamma_{23}<\gamma_{13}<\gamma_{44}<\gamma_{34} \cdots
$$

Let $A$ be the intersection matrix for the root system $\mathbb{A}_{n}$. The matrix $-A$ is negative definite, therefore there exists a unique vector $\bar{\alpha}=\left(\alpha_{i}\right)$ such that $(-A) \cdot \bar{\alpha}=\bar{\beta}$. If we now set $D_{0}=\sum_{i} \alpha_{i} E_{i}$ then $D_{0} \cdot e_{0}=0$ and $D_{0} \cdot e_{i}=\beta_{i}>0$ for $i=1, \ldots, n$; hence $D_{0}$ is in the relative interior of $\operatorname{Mov}(X) \cap e_{0}^{\perp}$. What is more, if we set $D_{t}=D_{0}-(t / 2) E_{0}$ then $D_{t} \cdot \lambda_{i j}=t-\gamma_{i j}$; so that $\gamma_{i j}$ is the threshold value of $t$ for the form $\lambda_{i j}$ on the half-line $\left\{D_{t}: t \in \mathbb{R}_{\geq 0}\right\}$. The SQM model of $X$ on which the divisor $D_{t}$ is ample will be denoted by $X_{t}$.

Now our theorem is equivalent to saying that the models $X_{t}$ are in bijection with connected components (open intervals) in $\mathbb{R}_{>0} \backslash\left\{\gamma_{i j}\right\}$. This can be verified by starting from the Hilb-Chow resolution associated with the interval $\left(0, \gamma_{11}\right)$ and proceeding inductively as it follows. Let $t$ be in the interval $\left(\gamma_{i j}, \gamma_{i^{\prime} j^{\prime}}\right)$, where $\gamma_{i j}$ and $\gamma_{i^{\prime} j^{\prime}}$ are consecutive numbers in the sequence of $\gamma^{\prime}$ 's. We verify first that the $\mathbb{P}^{2}$-s which are in the exceptional locus of $X_{t}$ have lines whose classes are only of type $\pm \lambda_{r s}$. Secondly, that pairs $(i, j)$ and $\left(i^{\prime}, j^{\prime}\right)$ are among those $(r, s)$ which occur on $X_{t}$. The sign of $\pm \lambda_{r s}$ will depend on the position of $\gamma_{r s}$ with respect to $t$. Hence we flop the $\mathbb{P}^{2}$ with lines of type $-\lambda_{i^{\prime} j^{\prime}}$ and proceed to the next interval. Note that with this single flop we keep the (relative) projectivity of the model (over $Y$ ). The argument will stop when $X_{t}$ contains only one $\mathbb{P}^{2}$, with lines in the class $+\lambda_{1 n}$. We run this algorithm for $n=7$ in the next section.

6.7. Explicit flops. The aim of the following diagrams is to describe explicitly the different SQM models obtained as resolutions of the 4-dimensional symplectic singularity coming from the action of $\mathbb{Z}_{n} \prec \mathbb{Z}_{2}$, for $n=7$ (or less). We use the set up and notation from Theorem 6.6 and its proof. In particular each SQM model corresponds to a connected component in $\mathbb{R}_{>0} \backslash\left\{\gamma_{i j}\right\}$, where $\gamma_{i j}$ are the threshold values associated with flops in 6.6.12 The diagram titled $\left(\gamma_{i j}, \gamma_{i^{\prime} j^{\prime}}\right)$ corresponds to the SQM model in the interval bounded by these thresholds. Each diagram 
describes the components of the special fiber of the resolution; these are the only things which vary from one model to the other. We actually omit the components which cannot become $\mathbb{P}^{2}$, i.e. the ones which will be never flopped and which are isomorphic to $F_{4}$ (see Lemma 6.4).

In each diagram a node corresponds to a component of the special fiber, i.e. to a surface. The isomorphism class of the surface is denoted by the following codes: $\boldsymbol{\Delta}=\mathbb{P}^{2}, \boldsymbol{\nabla}=F_{1}, \boldsymbol{\square}=\mathbb{P}^{1} \times \mathbb{P}^{1}$ and $\star$ denotes blow-up of $\mathbb{P}^{2}$ in two points (or $\mathbb{P}^{1} \times \mathbb{P}^{1}$ in one point).

The nodes of two intersecting components are connected by a line segment: this segment can be dotted, if the incidence between the components is a point, or solid, if the incidence is a curve. In fact, one can easily compute the cohomological classes of all incidence curves in our diagrams; we label only some of them for the sake of clarity of the picture. We use the classes in cohomology of essential curves, which, as above, we denote by $e_{0}, e_{1}, \ldots, e_{n}$, where $e_{0}$ is the class of a fiber over the surface of $\mathbb{A}_{1}$ singularities and $e_{1}, \ldots, e_{n}$ are components of the fiber over $\mathbb{A}_{n}$ singularity. Moreover, for $0 \leq i \leq j \leq n$, by $\lambda_{i j}$ we denote $e_{0}-\left(e_{i}+\cdots+e_{j}\right)$. In the diagrams below, for instance, $e_{i}$ 's appear as classes of rulings of quadrics as well as $F_{1}$ 's, while $\pm \lambda_{i j}$ 's are classes of lines in $\mathbb{P}^{2}$ or sections of $F_{1}$ 's. For example, incidence curves for $\mathbb{P}^{1} \times \mathbb{P}^{1}$, represented as edges of our diagrams at the vertex denoted by $\boldsymbol{\square}$, have the same classes at the opposite ends of the vertex: e.g. the class of $-\boldsymbol{m}$ is the same as of - . Also, if the class of $\rightarrow$ is $\lambda_{i j}$ and the class of $\leftarrow$ is $\lambda_{i+1, j}$ then the class of ruling, e.g. the class $\downarrow$, is equal to $\lambda_{i j}-\lambda_{i+1, j}=e_{i+1}$.

We think that the reader has now the necessary instructions to use the diagrams in order to take the "journey" through the different SQM models, following the path in $\operatorname{Mov}(X / Y)$ given, in the previous section, by the half-line $D_{0}+\mathbb{R}_{\geq 0} \cdot\left(-E_{0}\right)$. We can accompany the reader in the first steps: the first diagram represents the special fiber in the Hilb-Chow resolution, as described in 6.4. To go from this first model to the second, one has to cross the wall in $\operatorname{Mov}(X / Y)$ given by the hyperplane $\lambda_{11}^{\perp}$, that is one has to flop the $\mathbb{P}^{2}$ in the lower node, let us call it $(1,1)$. This will change the sign of the line of this $\mathbb{P}^{2}$ in the cohomology of $X$, from $-\lambda_{11}$ to $\lambda_{11}$. It will also change the component in the node $(1,2)$, from $\star$, the blow-up of $\mathbb{P}^{1} \times \mathbb{P}^{1}$ in one point, into $\downarrow$, which is $F_{1}$. The incidence between the components in the nodes $(1,1)$ and $(1,2)$ will change from a curve into a point. The step from the second to the third model consists in crossing the wall in $\operatorname{Mov}(X / Y)$ given by the hyperplane $\lambda_{22}^{\perp}$. This is the flop of the $\mathbb{P}^{2}$ in the node $(2,2)$. It will change the components in the node $(1,2),(1,3)$ and $(3,2)$ and their incidence, as indicated in the third diagram.

The journey will proceed in this way and it will end in the model described by the last diagram: here we have only one $\mathbb{P}^{2}$, which is in the node $(1,6)$, a bunch of $F_{1}$, in the nodes $(1, i)$ and $(j, 6), i \neq 6$ and $j \neq 1$, and $\mathbb{P}^{1} \times \mathbb{P}^{1}$ in the other nodes. 
$\left(0, \gamma_{11}\right)$

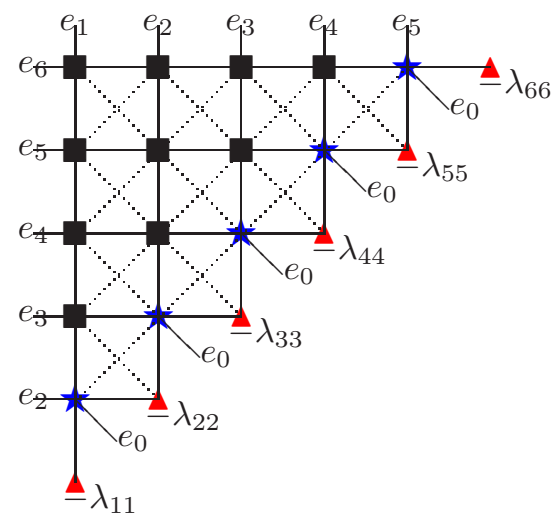

$\left(\gamma_{22}, \gamma_{12}\right)$

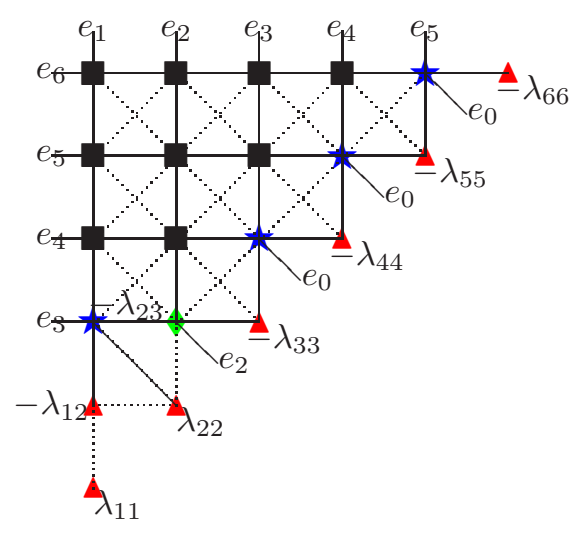

$\left(\gamma_{33}, \gamma_{23}\right)$

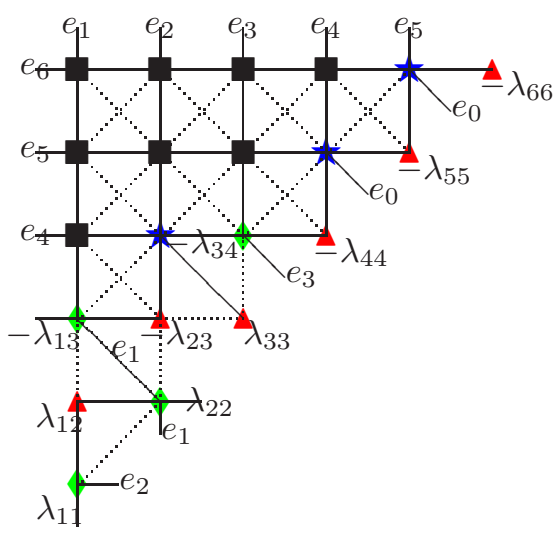

$\left(\gamma_{11}, \gamma_{22}\right)$

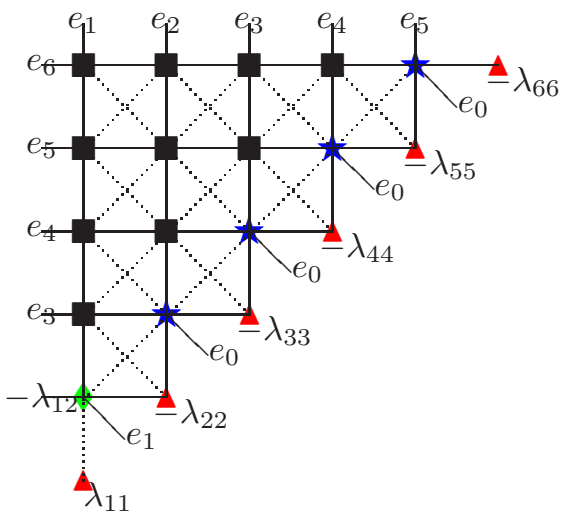

$\left(\gamma_{12}, \gamma_{33}\right)$

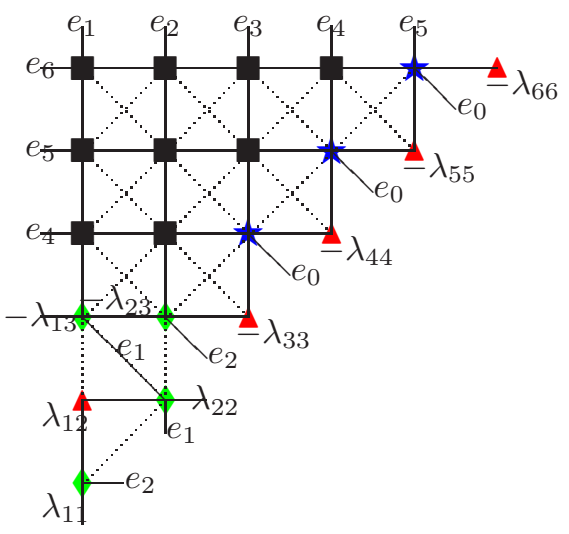

$\left(\gamma_{23}, \gamma_{13}\right)$

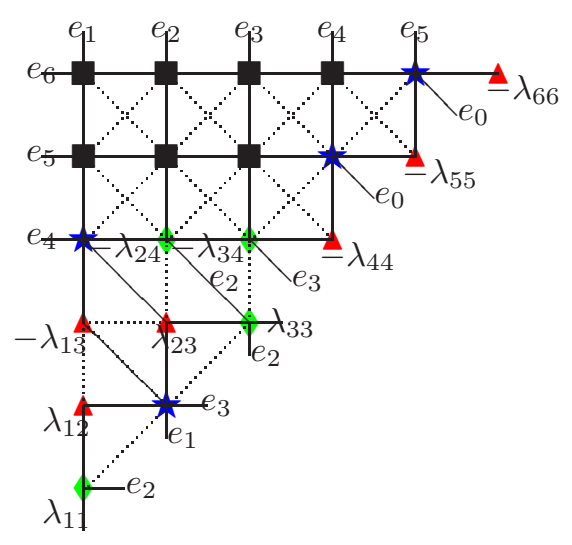



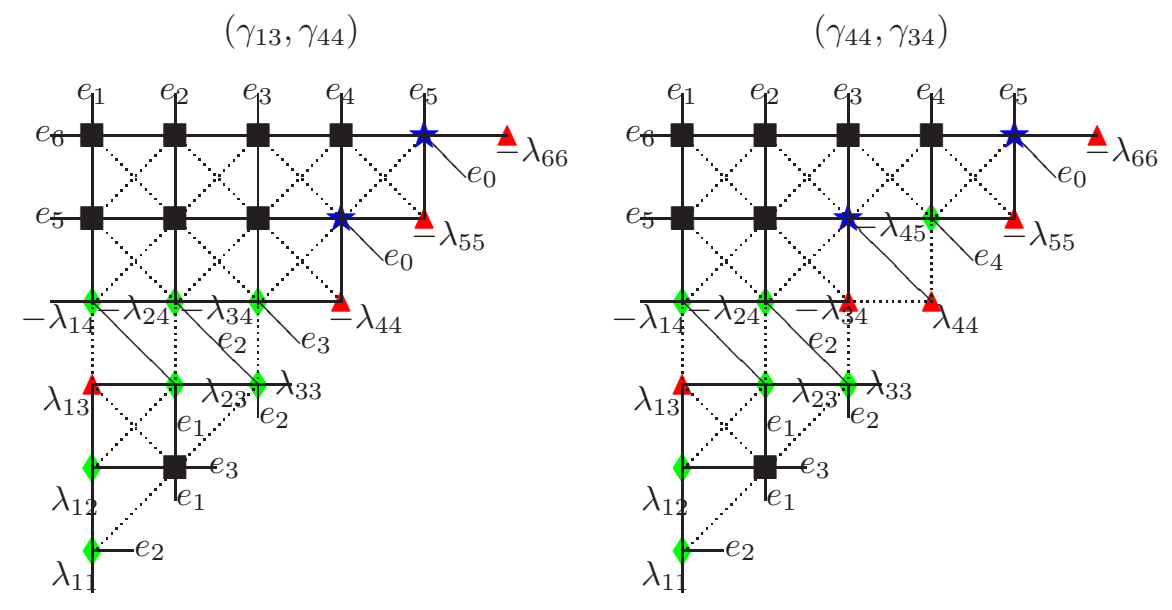

$\left(\gamma_{34}, \gamma_{24}\right)$

$\left(\gamma_{24}, \gamma_{14}\right)$
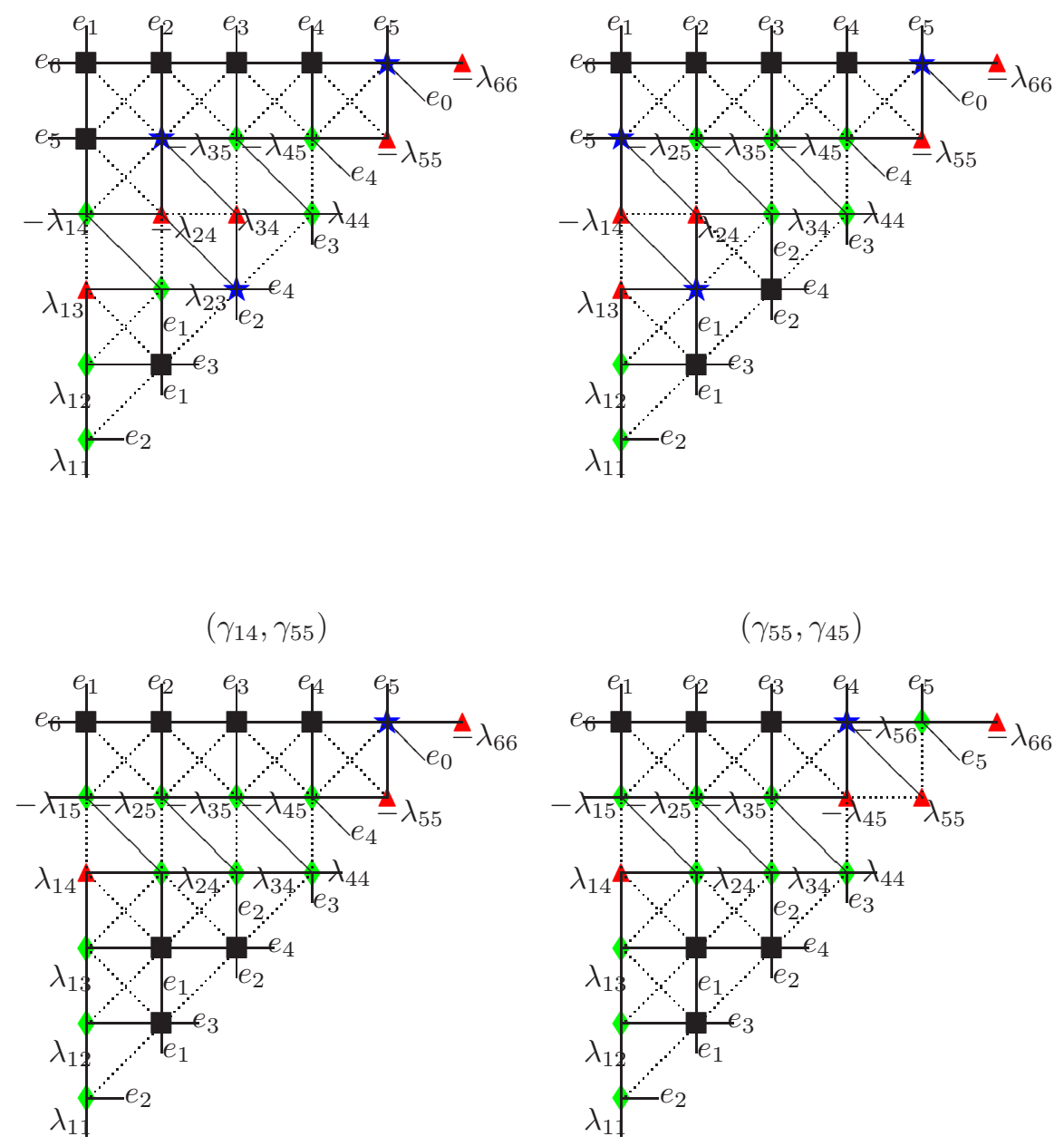

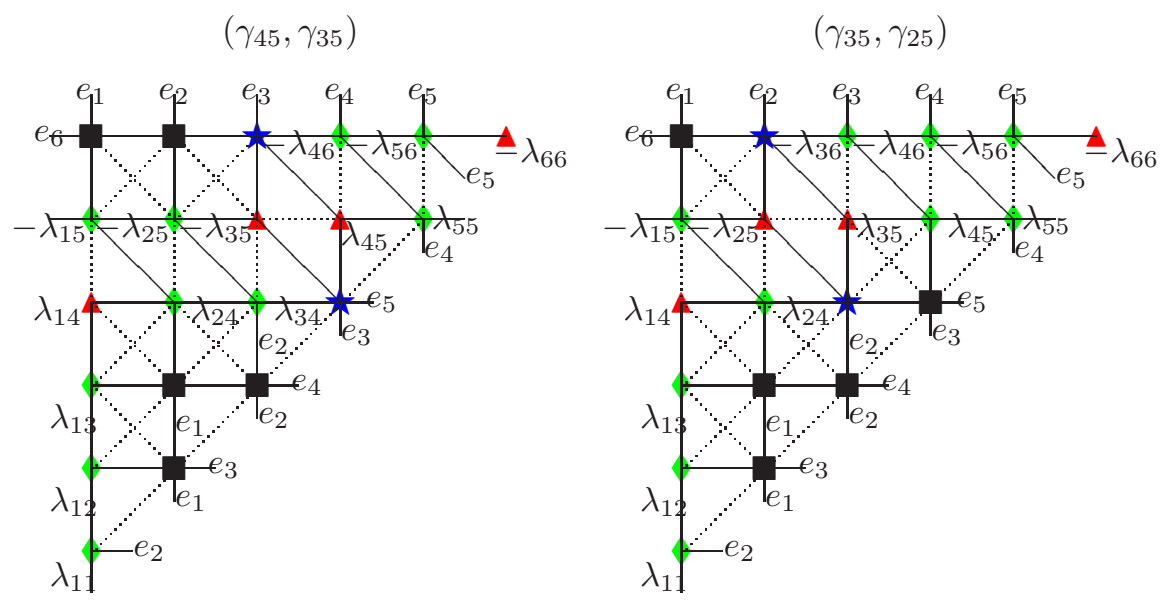

$\left(\gamma_{25}, \gamma_{15}\right)$
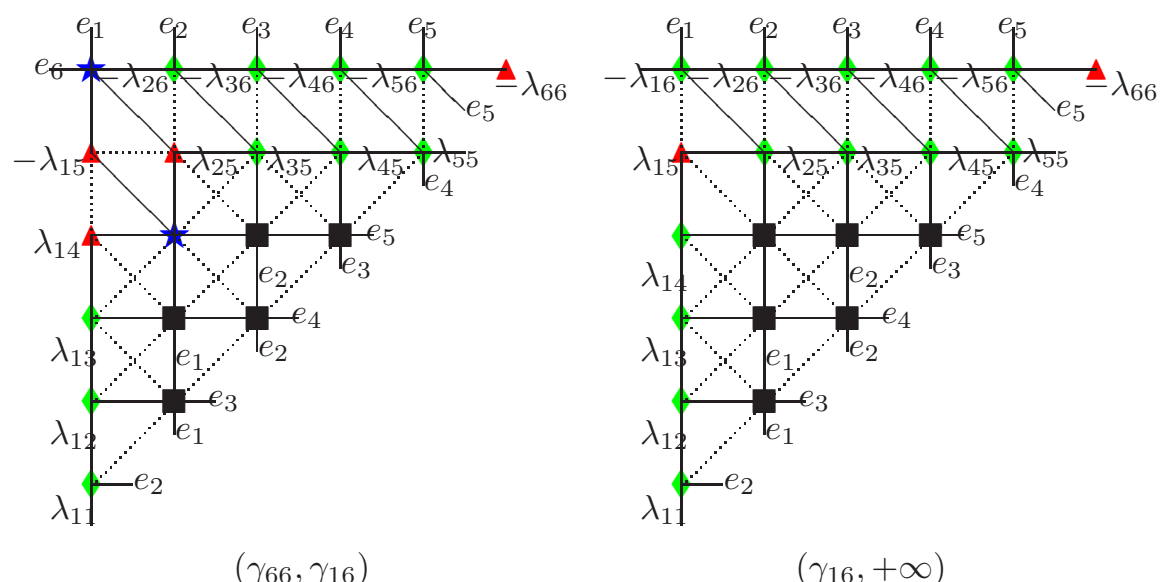

$\left.\gamma_{66}, \gamma_{16}\right)$

6 more flops in the upper row

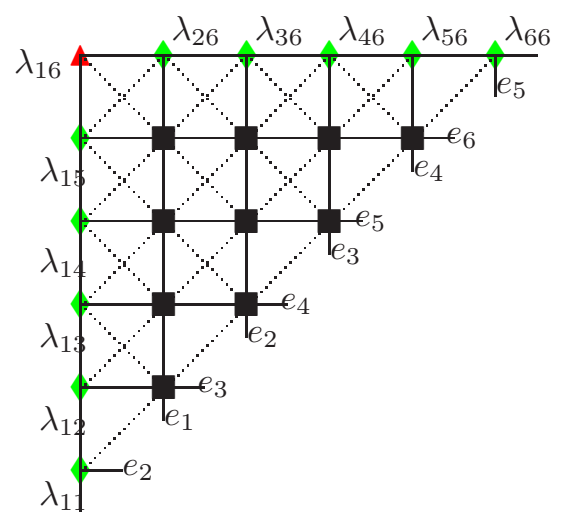

Let us note that similar diagrams are provided in Fu06a. The method used in that paper is similar to ours since the starting point is Hilb-Chow resolution but there each step involves several flops. However, the resulting diagrams in Fu06a are not quite correct since they imply that the components of the exceptional fiber in the final chamber are one $\mathbb{P}^{2}$ and all the rest $F_{1}$ 's. 


\section{Appendix}

7.1. Contraction to the nilpotent cone. In this section we recall known facts about flag varieties of simple Lie groups and about contractions to the nilpotent cone. This subject is classical and well documented, see e.g. [Slo80] or CM93] with the references therein. For a more recent survey see also Nam08. Our point of view is somehow more geometric, related to homogeneous varieties, in the spirit of Ott95, and directed on understanding the picture at the level of the related root systems. We refer to [TY05, Ch. 18] for generalities on root systems.

Let $G$ be a complex simple algebraic group with the Lie algebra $\mathfrak{g}$. By $R$ we denote the set of roots of $\mathfrak{g}$ and consider the lattices of roots and of weights $\Lambda_{R} \subset \Lambda_{W}$ of the algebra (or group) in question; let $V=\Lambda_{R} \otimes \mathbb{R}$. By $B$ we denote a Borel subgroup of $G$ and $F=G / B$ is its flag variety. It is known that we have a natural isomorphism $\operatorname{Pic} F \simeq \Lambda_{W}$ under which $\operatorname{Nef}(F) \subset N^{1}(F)$ is identified with the Weyl chamber in $V$. Under this identification any irreducible representation $U_{w}$ of $G$ with the highest weight $w$ corresponds to the complete linear system on $F$ of a nef line bundle whose associated map, $F \rightarrow \mathbb{P}\left(U_{w}\right)$, maps $F$ to the unique closed orbit. Moreover, the sum of the positive roots $\rho=\sum_{\alpha \in R^{+}} \alpha$ can be identified with the anticanonical class $-K_{F}$ and the Weyl formula, describing the dimension of irreducible representations, yields the Hilbert polynomial on Pic $F$. That is, for every $\lambda \in \Lambda_{W}$, the dimension formula, or the Euler characteristic of the respective line bundle on $F$, can be written as a polynomial

$$
H(\lambda)=\prod_{\alpha \in R^{+}} \frac{((\lambda+\rho / 2), \alpha)}{(\rho / 2, \alpha)}
$$

where $($,$) denotes the Killing form and R^{+}$is the set of positive roots. Note that the above polynomial is of degree equal to $\operatorname{dim} F ; H(-\lambda-\rho)=(-1)^{\operatorname{dim} F} H(\lambda)$ is exactly Serre duality.

The Killing form allows to relate $V$ to its dual. For every root $\alpha \in R$ we set $V^{*} \ni \alpha^{\vee}=(v \mapsto 2(\alpha, v) /(\alpha, \alpha))$. The facets of the Weyl chamber are supported by the simple roots, that is they are hypersurfaces defined by forms $\alpha^{\vee}$.

Lemma 7.1. The extremal contraction $\hat{\pi}_{\alpha}: F \rightarrow F_{\alpha}$ associated with the facet $\alpha^{\perp} \cap \operatorname{Nef}(F)$ is a $\mathbb{P}^{1}$ bundle and $\alpha^{\vee}$ is the class of the extremal curve in $N_{1}(F)$. In $\operatorname{Pic}(F)=\Lambda_{W}$ the class of the relative cotangent bundle, $\Omega\left(F / F_{\alpha}\right)$, is $-\alpha$.

Proof. Note that the restriction of the polynomial $H(\lambda)$ to the hyperplane $\alpha^{\perp}$ defined by the face $\alpha^{\vee}$ is of degree $\operatorname{dim} F-1$ and $\alpha^{\vee}(\rho)=2$, TY05, 18.7.6]. This means that the extremal contraction $F \rightarrow F_{\alpha}$ associated with the facet $\alpha^{\perp} \cap \operatorname{Nef}(F)$ is a $\mathbb{P}^{1}$ bundle and $\alpha^{\vee}$ is the class of the fiber. On the other hand, $\rho-\alpha \in \alpha^{\perp}$ and $\left.H\left(s_{\alpha}(\lambda)\right)-\alpha\right)=-H(\lambda)$, which is the relative duality

Let $X$ be the total space of the cotangent bundle of $F$, that is $X=\operatorname{Spec}_{F}(\operatorname{Symm}(T F))$. Recall that $T F=G \times_{B} \mathfrak{g} / \mathfrak{b}$, where $\mathfrak{b} \subset \mathfrak{g}$ is tangent to $B$ and $B$ acts on $\mathfrak{g} / \mathfrak{b}$ via adjoint representation and the quotient $\mathfrak{g} \rightarrow \mathfrak{g} / \mathfrak{b}$. Alternatively, $T^{*} F=G \times_{B} \mathfrak{u}$ where $\mathfrak{u} \subset \mathfrak{g}$ is the nilradical of $\mathfrak{b}$. The variety $X$ is symplectic.

Since $T F$ is spanned by its global sections, which form the Lie algebra $\mathfrak{g}$, we have a map $X \rightarrow \mathfrak{g}^{*}$ which contracts the zero section to 0 . The image is a normal variety called the nilpotent cone, which we denote it by $Y$. The map $\pi: X \rightarrow Y$ is a symplectic contraction. 
Clearly, $N^{1}(X / Y)=N^{1}(F), \operatorname{Nef}(X / Y)=\operatorname{Nef}(F)$ and every extremal contraction $\hat{\pi}_{\alpha}: F \rightarrow F_{\alpha}$, which is a $\mathbb{P}^{1}$ bundle, extends to a divisorial contraction $\pi_{\alpha}: X \rightarrow X_{\alpha}$ with all nontrivial fibers being $\mathbb{P}^{1}$. Let $E_{\alpha} \subset X$ be the exceptional divisor of $\pi_{\alpha}$ and $C_{\alpha}$ be a general fiber of $\pi_{\alpha}$ restricted to $E_{\alpha}$.

Lemma 7.2. The class of $C_{\alpha}$ in $V^{*}=N_{1}(X / Y)$ is $\alpha^{\vee}$. The class of $E_{\alpha}$ in Pic $X=\Lambda_{W}$ is $-\alpha$.

Proof. We have an exact sequence of vector bundles over $F$ :

$$
0 \longrightarrow \hat{\pi}_{\alpha}^{*}\left(\Omega F_{\alpha}\right) \longrightarrow \Omega F \longrightarrow \Omega\left(F / F_{\alpha}\right) \longrightarrow 0
$$

and the divisor $E_{\alpha}$ in the total space of $\Omega F$ is the total space of the sub-bundle $\pi_{\alpha}^{*}\left(\Omega F_{\alpha}\right)$. Thus, the restriction of its normal to $F$ is the line bundle $\Omega\left(F / F_{\alpha}\right)$ hence the lemma follows by 7.1 .

Corollary 7.3. c.f. Hin91, (5.2)] In the above situation, the intersection matrix $E_{\alpha} \cdot C_{\beta}$ is the negative of the Cartan matrix of the respective root system.

The above observation is the key for Brieskorn-Slodowy result on the type singularity of the nilpotent cone in of codimension 2; it can be expressed as follows:

Theorem 7.4. (Brieskorn, Slodowy) Let $\pi: X=G / B \rightarrow Y$ be the contraction to the nilpotent cone. If the root system of $G$ is of type $\mathbb{A}_{n}, \mathbb{D}_{n}, \mathbb{E}_{6}, \mathbb{E}_{7}, \mathbb{E}_{8}$ then in codimension 2 the contraction $\pi$ is the resolution of a surface Du Val singularity of the same $\mathbb{A}-\mathbb{D}-\mathbb{E}$ type. If $G$ is of type $\mathbb{B}_{n}, \mathbb{C}_{n}, \mathbb{F}_{4}$ and $\mathbb{G}_{2}$ then in codimension 2 the contraction $\pi$ is the resolution of singularities of type $\mathbb{A}_{2 n-1}, \mathbb{D}_{n+1}, \mathbb{E}_{6}$ and $\mathbb{D}_{4}$ and the irreducible components of the exceptional set of $\pi$ are in bijection with the orbits of the action of the group of automorphisms of the Dynkin diagrams of latter type.

We have the following immediate consequence of 7.1 and 7.2 .

Corollary 7.5. In the above case $\operatorname{Mov}(X / Y)=\operatorname{Nef}(X / Y)$ coincides with the Weyl chamber.

7.2. Resolving $\mathbb{C}^{4} / \sigma_{3}$. In this last section we will give an explicit description of the symplectic resolution of the quotient $\mathbb{C}^{4} / \sigma_{3}$ introduced in section 6.3 (see also AnWi10). We will constantly refer to the following commutative diagram, which comes from the presentation of $\sigma_{3}=D_{6}$ in terms of a semisimple product, $\sigma_{3}=\mathbb{Z}_{3} \rtimes \mathbb{Z}_{2}$ :

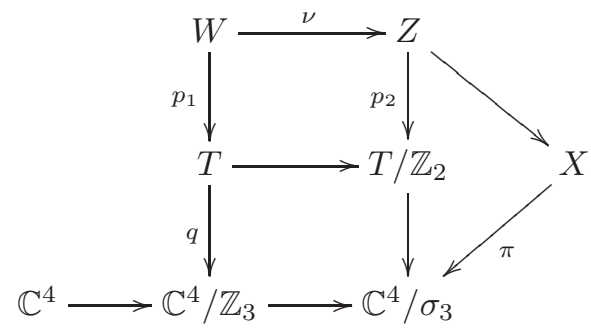

The vertical map $q: T \rightarrow \mathbb{C}^{4} / \mathbb{Z}_{3}$ is the toric resolution of $\mathbb{C}^{4} / \mathbb{Z}_{3}$ which can be described as follows. Let $N_{0}$ be a lattice with the basis $e_{1}, e_{2}, f_{1}, f_{2}$ and in $N_{0} \otimes \mathbb{R}$ take the standard cone $\left\langle e_{1}, e_{2}, f_{1}, f_{2}\right\rangle$ representing $\mathbb{C}^{4}$. The toric singularity $\mathbb{C}^{4} / \mathbb{Z}_{3}$ is obtained by extending $N_{0}$ to an overlattice $N$ (keeping the same cone) generated 
by adding to $N_{0}$ an extra generator $v_{1}=\left(e_{1}+e_{2}\right) / 3+2\left(f_{1}+f_{2}\right) / 3$. If $v_{2}=$ $2\left(e_{1}+e_{2}\right) / 3+\left(f_{1}+f_{2}\right) / 3$ then the rays generated by $e_{i}$ 's, $f_{i}$ 's and $v_{i}$ 's are in the fan of the toric resolution of $\mathbb{C}^{4} / \mathbb{Z}_{3}$ which is presented in the following picture by taking a affine hyperplane section of the cone $\left\langle e_{1}, e_{2}, f_{1}, f_{2}\right\rangle$. The solid edges are the boundary of the cone while its division is marked by dotted line segments.

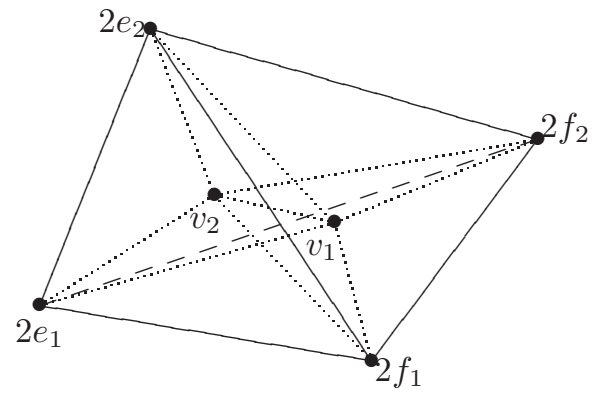

The exceptional set of this resolution consists of two divisors, $E_{1}, E_{2}$, both isomorphic to a $\mathbb{P}^{2}$ - bundle over $\mathbb{P}^{1}$, namely $\mathbb{P}(\mathcal{O}(2) \oplus \mathcal{O} \oplus \mathcal{O})$. They intersect along a smooth quadric $\mathbb{P}^{1} \times \mathbb{P}^{1}$.

The action of $\mathbb{Z}_{2}$ on $\mathbb{C}^{4} / \mathbb{Z}_{3}$ can be lifted up to an action on $T$. This action, which is induced by the reflections in $\sigma_{3}=D_{6}$, identifies the two divisors by identifying the $\mathbb{P}^{2}$ ruling of $E_{1}$ with that of $E_{2}$; on the intersection the action interchanges the coordinates on $\mathbb{P}^{1} \times \mathbb{P}^{1}$.

Going back to the diagram 7.2.13 $p_{2}$ is the resolution of the quotient $T / \mathbb{Z}_{2}$ obtained by blowing up the surface which is the locus of $A_{1}$-singularities. The morphism $p_{1}$ is the blow-up along the fixed point set of the $\mathbb{Z}_{2}$-action. We denote by $\Delta_{W}$ and $\Delta_{Z}$ the exceptional divisors. Then $\nu$ is a $2: 1$ cover ramified along $\Delta_{W}$.

The divisor $\Delta_{W}$ is irreducible and its intersection with the fiber over the special point, which is the strict transformof $E_{1} \cup E_{2}$, call it $E_{1}^{\prime} \cup E_{2}^{\prime}$, is equal to the 3rd Hirzebruch surface $F_{3}$. This follows from computing the normal of the curve which is the fixed point set of the $\mathbb{Z}_{2}$ action in the exceptional locus of $T$. Indeed, the normal of the intersection $E_{1} \cap E_{2}=\mathbb{P}^{1} \times \mathbb{P}^{1}$ is $\mathcal{O}(1,-2)+\mathcal{O}(-2,1)$ and the normal of the diagonal in the intersection is $\mathcal{O}(2)$. Thus the normal of the diagonal of $\mathbb{P}^{1} \times \mathbb{P}^{1}$ in $T$ is $\mathcal{O}(-1) \oplus \mathcal{O}(-1) \oplus \mathcal{O}(2)$ and, since its normal in the fixed point set is $\mathcal{O}(-1)$, it follows that the normal of the fixed point set over the diagonal is $\mathcal{O}(-1) \oplus \mathcal{O}(2)$. Finally, let us note that the intersection in $W$ of the $F_{3}$ surface with the strict transform of $\mathbb{P}^{1} \times \mathbb{P}^{1}$ is the exceptional curve in the surface $F_{3}$ and the diagonal in $\mathbb{P}^{1} \times \mathbb{P}^{1}$.

The fibers of the ruling $E_{i} \rightarrow \mathbb{P}^{1}$, for $i=1,2$, are blown up in $E_{i}^{\prime}$ to ruled surfaces (1st Hirzebruch) and the map $E_{i}^{\prime} \rightarrow \mathbb{P}^{1}$ can be factored either by blow down $E_{i}^{\prime} \rightarrow E_{i}$ or by a $\mathbb{P}^{1}$-bundle $E_{i}^{\prime} \rightarrow F_{3}$.

The strict transform of the surface $E_{1} \cap E_{2}=\mathbb{P}^{1} \times \mathbb{P}^{1}$ is mapped via the quotient map $W \rightarrow Z$ to $\mathbb{P}^{2}$, and this is a double covering ramified over the diagonal in $\mathbb{P}^{1} \times \mathbb{P}^{1}$. The exceptional curve of $F_{3}$, which is the diagonal in $\mathbb{P}^{1} \times \mathbb{P}^{1}$, becomes a conic in $\mathbb{P}^{2}$. Thus, eventually, we see that $E_{1}^{\prime}$ is identified with $E_{2}^{\prime}$ and via $\nu$ they are sent to a (non-normal) divisor $E_{Z}$ in $Z$. The divisors $\Delta_{W}$ and $E_{Z}$ generate Pic $Z$ and $K_{Z}=E_{Z}$.

From the computation of the intersection of curves and divisors we see that the divisor $E_{Z}$ is not numerically effective, hence $Z$ admits a birational Fano-Mori contraction $Z \rightarrow X$ with exceptional divisor $E_{Z}$. We describe the contraction by 
looking at the normalization of $E_{Z}$. Namely, by looking at the numerical classes of curves, we conclude that the resulting map is a composition $E_{1}^{\prime} \rightarrow F_{3} \rightarrow S_{3}$, where the latter map is the contraction of the exceptional curve in $F_{3}$ to the vertex of the cubic cone $S_{3}$. Therefore a general fiber of $Z \rightarrow X$ over $E_{Z}$ is a $\mathbb{P}^{1}$ - that is, generally this is a blow-down of the divisor $E_{Z}$ to a surface - while the special fiber is a $\mathbb{P}^{2}$. Such a contraction was discussed in AnWi98 where it was proved that the image $X$ is a smooth 4 -fold and the divisor $E_{Z} \subset Z$ is blow-down to the rational cubic cone $S_{3} \subset X$. Moreover $K_{X}=\mathcal{O}_{X}$.

Let us finally consider the induced map $\pi: X \rightarrow Y:=\mathbb{C}^{4} / \sigma_{3}$. It is a crepant contraction which contracts the divisor $\Delta_{W}$ to a surface $S$ which, outside the point 0 , is a smooth surface of $A_{1}$ singularities (coming from the $\mathbb{Z}_{2}$-action); moreover it contracts $S_{3}$ to 0 . The surface $S$ is non-normal in 0 . This is a crepant, hence symplectic, resolution of $\mathbb{C}^{4} / \sigma_{3}$.

Note that $\operatorname{Pic}(X / Y)=\mathbb{Z}$, therefore $\operatorname{Mov}(X / Y)$ is one dimensional. This is the only SQM model over $Y$.

We conclude with the description of the family of rational curves (of $X$ over $Y$ ). Let $C$ be the essential curve of the symplectic resolution $\pi: X \rightarrow Y:=\mathbb{C}^{4} / \sigma_{3}$ and let $\mathcal{V} \subset$ RatCurves $^{n}(X / Y)$ be a family containing $C$. Then $\mathcal{V}$ is a smooth surface which contains a (-1)-curve, which parametrizes the lines in the ruling of $S_{3}$. The normalization of $S$ is a smooth surface and $\mathcal{V}$ is obtained by blowing up the point of the normalization which stays over 0 .

\section{REFERENCES}

[ADHL10] Ivan Arzhantsev, Ulrich Derenthal, Juergen Hausen, and Antonio Laface. Cox rings. arXiv:math/1003.4229 [math.AG], 2010.

[AnWi98] Marco Andreatta and Jarosław A. Wiśniewski. On contractions of smooth varieties. $J$. Algebraic Geom., 7(2):253-312, 1998.

[AnWi10] Marco Andreatta and Jarosław A. Wiśniewski. On the Kummer construction. Rev. Mat. Complut., 23(1):191-215, 2010.

[AlWi13] Klaus Altmann and Jarosław Wiśniewski. Essential curves and Fano spaces. In preparation, 2013.

[Bea83] Arnaud Beauville. Variétés Kähleriennes dont la première classe de Chern est nulle. J. Differential Geom., 18(4):755-782 (1984), 1983.

[Bea00] Arnaud Beauville. Symplectic singularities. Invent. Math., 139(3):541-549, 2000.

[Bel09] Gwyn Bellamy. On singular Calogero-Moser spaces. Bull. Lond. Math. Soc., 41(2):315326, 2009.

[BHL03] Dan Burns, Yi Hu, and Tie Luo. HyperKähler manifolds and birational transformations in dimension 4. In Vector bundles and representation theory (Columbia, MO, 2002), volume 322 of Contemp. Math., pages 141-149. Amer. Math. Soc., Providence, RI, 2003.

[Bou75] N. Bourbaki. Éléments de mathématique. Hermann, Paris, 1975. Fasc. XXXVIII: Groupes et algèbres de Lie. Chapitre VII: Sous-algèbres de Cartan, éléments réguliers. Chapitre VIII: Algèbres de Lie semi-simples déployées, Actualités Scientifiques et Industrielles, No. 1364.

[CM93] David H. Collingwood and William M. McGovern. Nilpotent orbits in semisimple Lie algebras. Van Nostrand Reinhold Mathematics Series. Van Nostrand Reinhold Co., New York, 1993.

[FN04] Baohua Fu and Yoshinori Namikawa. Uniqueness of crepant resolutions and symplectic singularities. Ann. Inst. Fourier (Grenoble), 54(1):1-19, 2004.

[Fog68] John Fogarty. Algebraic families on an algebraic surface. Amer. J. Math, 90:511-521, 1968.

[Fu06a] Baohua Fu. Mukai flops and deformations of symplectic resolutions. Math. Z., 253(1):87-96, 2006. 
[Fu06b] Baohua Fu. A survey on symplectic singularities and symplectic resolutions. Ann. Math. Blaise Pascal, 13(2):209-236, 2006.

[GK04] Victor Ginzburg and Dmitry Kaledin. Poisson deformations of symplectic quotient singularities. Adv. Math., 186(1):1-57, 2004.

[Hin91] V. Hinich. On Brieskorn's theorem. Israel J. Math., 76(1-2):153-160, 1991.

[HK00] Yi Hu and Sean Keel. Mori dream spaces and GIT. Michigan Math. J., 48:331-348, 2000. Dedicated to William Fulton on the occasion of his 60th birthday.

[HT09] Brendan Hassett and Yuri Tschinkel. Moving and ample cones of holomorphic symplectic fourfolds. Geom. Funct. Anal., 19(4):1065-1080, 2009.

[Kal02] D. Kaledin. McKay correspondence for symplectic quotient singularities. Invent. Math., 148(1):151-175, 2002.

[Kal03] D. Kaledin. On crepant resolutions of symplectic quotient singularities. Selecta Math. (N.S.), 9(4):529-555, 2003.

[Kal06] D. Kaledin. Symplectic singularities from the Poisson point of view. J. Reine Angew. Math., 600:135-156, 2006.

[KaMa87] Yujiro Kawamata and Kenji Matsuki. The number of the minimal models for a 3-fold of general type is finite. Math. Ann., 276(4):595-598, 1987.

[KoMo98] János Kollár and Shigefumi Mori. Birational geometry of algebraic varieties, volume 134 of Cambridge Tracts in Mathematics. Cambridge University Press, Cambridge, 1998. With the collaboration of C. H. Clemens and A. Corti, Translated from the 1998 Japanese original.

[KMM87] Yujiro Kawamata, Katsumi Matsuda, and Kenji Matsuki. Introduction to the minimal model problem. In Algebraic geometry, Sendai, 1985, volume 10 of Adv. Stud. Pure Math., pages 283-360. North-Holland, Amsterdam, 1987.

[Kol96] János Kollár. Rational curves on algebraic varieties, volume 32 of Ergebnisse der Mathematik und ihrer Grenzgebiete. 3. Folge. A Series of Modern Surveys in Mathematics [Results in Mathematics and Related Areas. 3rd Series. A Series of Modern Surveys in Mathematics]. Springer-Verlag, Berlin, 1996.

[LS08] Manfred Lehn and Christoph Sorger. A symplectic resolution for the binary tetrahedral group. arXiv:0810.3225 [math.AG], 2008.

[Mat91] Kenji Matsuki. Termination of flops for 4-folds. Amer. J. Math., 113(5):835-859, 1991.

[Nam01] Yoshinori Namikawa. Extension of 2-forms and symplectic varieties. J. Reine Angew. Math., 539:123-147, 2001.

[Nam08] Yoshinori Namikawa. Birational algebraic geometry and nilpotent orbits. Sūgaku, 60(3):295-318, 2008.

[Ott95] Giorgio Ottaviani. Rational homogeneous varities, Cortona course notes, 1995. http://web.math.unifi.it/users/ottavian/rathomo/rathomo.pdf. 1995.

[SCW04] Luis Eduardo Solá Conde and Jarosław A. Wiśniewski. On manifolds whose tangent bundle is big and 1-ample. Proc. London Math. Soc. (3), 89(2):273-290, 2004

[Slo80] Peter Slodowy. Simple singularities and simple algebraic groups, volume 815 of Lecture Notes in Mathematics. Springer, Berlin, 1980.

[TY05] Patrice Tauvel and Rupert W. T. Yu. Lie algebras and algebraic groups. Springer Monographs in Mathematics. Springer-Verlag, Berlin, 2005.

[Ver00] Misha Verbitsky. Holomorphic symplectic geometry and orbifold singularities. Asian J. Math., 4(3):553-563, 2000.

[Wie02] Jan Wierzba. Birational geometry of symplectic 4-folds. http://www.mimuw.edu.pl/ jarekw/postscript/bir4fd.ps, 2002.

[Wie03] Jan Wierzba. Contractions of symplectic varieties. J. Algebraic Geom., 12(3):507-534, 2003.

[WW03] Jan Wierzba and Jarosław A. Wiśniewski. Small contractions of symplectic 4-folds. Duke Math. J., 120(1):65-95, 2003.

Dipartimento di Matematica, Universita di Trento, I-38050 Povo (TN)

E-mail address: marco.andreatta@unitn.it

Instytut Matematyki UW, Banacha 2, PL-02097 Warszawa

E-mail address: J.Wisniewski@mimuw.edu.pl 